\title{
A Modal Characterization of Alternating Approximate Bisimilarity
}

\author{
Jinjin Zhang • Zhaohui Zhu
}

Received: date / Accepted: date

\begin{abstract}
Recently, alternating transition systems are adopted to describe control systems with disturbances and their finite abstract systems. In order to capture the equivalence relation between these systems, a notion of alternating approximate bisimilarity is introduced. This paper aims to establish a modal characterization for alternating approximate bisimilarity. Moreover, based on this result, we provide a link between specifications satisfied by the samples of control systems with disturbances and their finite abstractions.
\end{abstract}

Keywords Alternating approximate bisimilarity · modal characterization · control systems with disturbances · finite abstractions · temporal logical specification

\section{Introduction}

The notion of bisimilarity is one of the central concepts in process algebra. Roughly speaking, two states are bisimilar if and only if they can perform same actions to reach bisimilar states. In general, two bisimilar processes are always considered to be identical. In recent years, the notion of bisimilarity has

This work received financial support of the National Natural Science of China(No. 60973045), Fok Ying-Tung Education Foundation, the NSF of Jiangsu Province (No.BK2012473) and the PAPD of JiangSu Higher Education Institutions (Audit Science and Technology Preliminary Research Project (No.YSXKKT27)) .

Jinjin Zhang

School of Information Science, Nanjing Audit University, Nanjing, 211815 China

Tel. +86-13770658202

E-mail: jinjinzhang@nau.edu.cn

Zhaohui Zhu: Corresponding author

Department of Computer Science, Nanjing University of Aeronautics and Astronautics, Nanjing, 210016 China

E-mail: bnj4892856@jlonline.com 
been adopted in the area of control theory to capture the equivalence between control systems and their finite abstraction [28] 29] 30].

However, when the states or actions of labeled transition systems are associated with quantitative data, the notion of bisimilarity seems not be very suitable for describing the equivalence in such situation. For example, in real time systems, there is often a little difference between time delays. If we use the usual notion of bisimilarity (for instance, timed bisimilarity [22] 31]) to capture the equivalence of states in such systems, time delays can match only if they are identical. Such exact matching may be unrealistic. On the other hand, in control theory, it has been pointed out that the notion of bisimilarity is so rigorous that it is often hard to construct finite abstractions which are bisimilar to the given control systems [14]24.

To overcome these defects, a number of theories are provided to describe approximate behavioral equivalence [5] 6] 7] 10, 11, 13, 15] 25] 26, 32. In these work, two different approaches have been adopted.

One approach is to introduce notions of approximate bisimilarity. In such category, Giacalone et al. are probably first to present the notion of approximate bisimilarity, and provide $\varepsilon$-bisimilarity for probabilistic transition systems [13. In the framework of metric labeled transition systems, Ying provides the notion of $\lambda$-bisimilarity [32. This notion has been adopted to describe the equivalence between processes in time-CCS and time-CSP [32, the equivalence and reliability of processes in pi-calculus with noise [33, and the equivalence between quantum processes in qCCS 34.

In recent years, some notions of approximate bisimilarity are introduced in control theory. In the framework of LTS with observations and metrics over observations, Girard and Pappas introduce $\delta$-approximate bisimilarity [15]. Pola and Tabuada adopt this notion to capture the equivalence between control systems without disturbances and their finite abstractions [24]. They also provide the notion of alternating approximate bisimilarity in alternating transition systems to describe approximate equivalence between control systems with disturbances and their finite abstractions 25] 26]. The notions of approximate bisimilarity play an important role in the analysis and design of control systems (for example, see [8] 27]). Girard and Pappas give an overview about the related work and point out that the notions of approximate bisimilarity provide a bridge between control theory and computer science [17.

Another approach is based on distance functions over processes (or states, systems). For a variety of transition systems, distance functions have been introduced via distinct approaches (e.g., modal logic, fixed point, and coalgebra). For example, for probabilistic transition systems, Desharnais et al. [10] 11 and Breugel et al. 6] [7] adopt these methods to define metrics over processes and establish the relationship between these metrics. Recently, Zhou and Ying define a metric over probabilistic transition systems in terms of so-called "smallest" logical formula that distinguishes them [38. For labeled transition systems accompanied with metric, van Breugel provides pseudometrics over states through these three methods and shows that these pseudometrics coincide [5]. 
To describe the equivalence between metric transition systems, de Alfaro et al. introduce linear distances and branching distances [9].

The relationship between these two approaches has been explored in the literature. For example, Giacalone et al. introduce a pseudometric over probabilistic transition systems in terms of $\varepsilon$-bisimilarity [13. Van Breugel presents a conjecture which concerns the relationship between his behavioural pseudometric and Ying's $\lambda$-bisimilarity [5]. Recently, a negative answer to this conjecture is given [36. In the framework of LTS with observations, Girard and Pappas characterize the branching distance in terms of $\delta$-approximate bisimulation with the assumption that the discount factor $\alpha=1$ [15]. This result has been generalized to general case and the branching distance with arbitrary discount factor is characterized in terms of $(\eta, \alpha)$-bisimilarity [37.

As well known, bisimilarity can be characterized as a fixed point [23, via a modal logic [18] and by way of coalgebra [1]. A modal characterization of bisimilarity is provided by Hennessy and Milner 18. They demonstrate that, in the framework of LTS, bisimilarity coincides with logical equivalence w.r.t Hennessy-Milner logic (HML, for short), that is, two states in LTS are bisimilar if and only if they satisfy the same formulae of HML. Inspired by this result, different modal characterizations are established for a lot of varieties of bisimilarity in the above style. For instance, Alur et al. characterize alternating bisimilarity in terms of alternating-time temporal logic (ATL, for short) 3 . Ying provides a logical characterization of $\lambda$-bisimilarity with the assumption that the metric is ultra-metric or $\lambda=0$. However, without such assumption, $\lambda$-bisimilarity can not be characterized in the style of Hennessy-Milner theorem. Its logical characterization associated with arbitrary metric is established in a new style 35 .

The logical characterizations of bisimilarity play important roles in the formal analysis and design of control systems. They guarantee that control systems share the same logical properties with their finite abstractions which are bisimilar to these control systems. In such situation, the analysis and design of control systems can be equivalently performed on their finite abstraction, which considerably reduces the complexity of the analysis and design of control systems 4,30 .

This paper aims to establish a logical characterization of alternating approximate bisimilarity. Furthermore, based on this result, for control systems with disturbances mentioned in [26, we illustrate a relationship between linear temporal logical specifications satisfied by their samples under control and by their finite abstractions under control, respectively. Roughly speaking, this paper demonstrates that if the sample of a control system with disturbances and its finite abstraction are alternating approximate bisimilar and the latter satisfies a specification under control, then the former may satisfy a "looser" specification under control. In particular, the transformation from a given specification to a looser one is provided.

The rest of this paper is organized as follows. We recall related definitions and results in Section 2. In Section 3. we provide a variety of ATL and two relations between the formulas of this logical language, which play central roles 
in this paper. Section 4 establishes a modal characterization of alternating approximate bisimilarity. In Section 5, we illustrate a relationship between temporal logical specifications satisfied by the samples of control systems with disturbances and by their finite abstractions under control. Finally, we conclude the paper in Section 6.

\section{Preliminaries}

This section will recall some notions and results about alternating transition systems, alternating bisimilarity and alternating approximate bisimilarity from [3] [26].

Before doing so, we introduce some useful notations. The symbol $\mathbb{N}, \mathbb{R}, \mathbb{R}_{+}$ and $\mathbb{R}_{+}^{0}$ denote the set of positive integers, reals, positive reals and nonnegative reals, respectively. For any set $A, A^{+}$represents the set of all non-empty finite strings over $A$, and $A^{\omega}$ denotes the set of infinite strings over $A$. We use $s_{A}$ and $\sigma_{A}$ to denote the elements of $A^{+}$and $A^{\omega}$, respectively. If $A$ is known from the context, we will omit the subscripts in $s_{A}$ and $\sigma_{A}$. For any $s \in A^{+}, s[i]$ and $s[$ end $]$ mean the $i$-th element and the last element of $s$, respectively. Given $i \leq$ $j, s[i, j], s[i, e n d]$ and $\sigma[i, \infty]$ represent $s[i] s[i+1] \cdots s[j], s[i] s[i+1] \cdots s[$ end $]$ and $\sigma[i] \sigma[i+1] \cdots$, respectively. As usual, $|s|$ means the length of $s$.

\subsection{Alternating transition systems}

Definition 1 An alternating transition system is a 5 -tuple $(S, \mathbb{P}, \Omega, \Pi, \hbar)$, where

- $S$ is a set of states;

- $\mathbb{P}$ is a set of observations;

- $\Omega$ is a finite set of agents;

- $\Pi: S \rightarrow \mathbb{P}$ is an observation function;

- $\hbar: S \times \Omega \rightarrow 2^{2^{S}}$ is a function satisfying that for any state $q \in S$, if each agent $i \in \Omega$ chooses a set $S_{i} \in \hbar(q, i)$, then the set $\bigcap_{i \in \Omega} S_{i}$ is a singleton. The function $\hbar$ is often said to be transition function.

If $\bigcup \hbar(q, i)$ is finite for each $q \in S$ and $i \in \Omega$, then we say $(S, \mathbb{P}, \Omega, \Pi, \hbar)$ is finite branching. If both the state set $S$ and the observation set $\mathbb{P}$ are finite, then $(S, \mathbb{P}, \Omega, \Pi, \hbar)$ is said to be a finite alternating transition system.

Intuitively, for each state $q$, an agent $i$ can choose a set $S_{i} \in \hbar(q, i)$ such that the state reached from $q$ must belong to $S_{i}$. According to the above definition, it is clear that the successor state of state $q$ is determined when all agents make choices.

Definition 2 Let $T=(S, \mathbb{P}, \Omega, \Pi, \hbar)$ be an alternating transition system, $i \in \Omega$ and $A g \subseteq \Omega$. A function $f_{i}: S^{+} \rightarrow 2^{S}$ is said to be a strategy of $i$ iff $f_{i}(s) \in \hbar(s[e n d], i)$ for any $s \in S^{+}$. A function $F_{A g}: S^{+} \rightarrow 2^{S}$ is said to be a strategy of $A g$ iff there exist a family of strategies $f_{i}$ of $i(i \in A g)$ such that $F_{A g}(s)=\bigcap_{i \in A g} f_{i}(s)$ for any $s \in S^{+}$. 
In the following, we set $\hbar(q, A g) \triangleq\left\{\bigcap_{i \in A g} S_{i}: S_{i} \in \hbar(q, i)\right.$ for each $\left.i \in A g\right\}$ for each state $q$ and agent set $A g$. The conclusion below is simple but useful.

Lemma 1 Let $T=(S, \mathbb{P}, \Omega, \Pi, \hbar)$ be an alternating transition system and $A g \subseteq \Omega$. The function $F_{A g}: S^{+} \rightarrow 2^{S}$ is a strategy of $A g$ if and only if $F_{A g}(s) \in \hbar(s[$ end $], A g)$ for any $s \in S^{+}$.

Proof (From Left to Right) Immediately follows from Definition 2 and the definition of $\hbar$.

(From Right to Left) Suppose that the function $F_{A g}: S^{+} \rightarrow 2^{S}$ satisfies that $F_{A g}(s) \in \hbar(s[e n d], A g)$ for any $s \in S^{+}$. To show that this function is a strategy of $A g$, construct a family of strategies $f_{i}(i \in A g)$ as follows:

Let $s \in S^{+}$. Since $F_{A g}(s) \in \hbar(s[e n d], A g)$, it follows from the definition of $\hbar(s[e n d], A g)$ that there exist $Q_{i} \in \hbar(s[e n d], i)$ for each $i \in A g$ such that $F_{A g}(s)=\bigcap_{i \in A g} Q_{i}$. Then, fix these $Q_{i}$ 's, and for each $i \in A g$, we set $f_{i}(s)=$ $Q_{i}$.

Clearly, by Definition 2 for each $i \in A g$, the function $f_{i}: S^{+} \rightarrow 2^{S}$ defined above is a strategy of $i$. On the other hand, it is easy to check that for any $s \in S^{+}, F_{A g}(s)=\bigcap_{i \in A g} f_{i}(s)$. Therefore, it follows from Definition 2 that $F_{A g}$ is a strategy of $\mathrm{Ag}$.

In general, the strategies are provided for some agents to enforce the outcomes of alternating transition systems to satisfy the given properties, such as reachability, safety and so on. Formally, the outcomes of alternating transition systems under strategies are defined below.

Definition 3 Let $T=(S, \mathbb{P}, \Omega, \Pi, \hbar)$ be an alternating transition system, $q \in S, A g \subseteq \Omega$ and let $F_{A g}: S^{+} \rightarrow 2^{S}$ be a strategy of $A g$. For each $n \in \mathbb{N}$,

$$
\begin{aligned}
& O u t_{T}^{n}\left(q, F_{A g}\right) \triangleq\left\{s \in S^{+}: s[1]=q,|s|=n\right. \text { and } \\
& \left.\quad \forall i<n \exists Q \in \hbar(s[i], \Omega-A g)\left(F_{A g}(s[1, i]) \cap Q=\{s[i+1]\}\right)\right\}
\end{aligned}
$$

and

$$
\begin{aligned}
& \operatorname{Out}_{T}\left(q, F_{A g}\right) \triangleq\left\{\sigma \in S^{\omega}\right.: \sigma[1]=q \text { and } \\
&\left.\forall i \in \mathbb{N} \exists Q \in \hbar(\sigma[i], \Omega-A g)\left(F_{A g}(\sigma[1, i]) \cap Q=\{\sigma[i+1]\}\right)\right\} .
\end{aligned}
$$

Intuitively, $F_{A g}$ is used to indicate a family of choices of agent set $\mathrm{Ag}$, while $\operatorname{Out}_{T}^{n}\left(q, F_{A g}\right)$ and $\operatorname{Out}_{T}\left(q, F_{A g}\right)$ consist of finite and infinite traces starting from $q$ in which each step subjects to such choices. We often omit the subscripts of $O u t_{T}^{n}\left(q, F_{A g}\right)$ and $\operatorname{Out}_{T}\left(q, F_{A g}\right)$ when the alternating transition system $T$ is clear from the context.

Lemma 2 For any $n \in \mathbb{N}$, the following conclusion holds:

$$
\begin{aligned}
\operatorname{Out}_{T}^{n+1}\left(q, F_{A g}\right)= & \left\{s \in S^{n+1}: s[1, n] \in O u t_{T}^{n}\left(q, F_{A g}\right)\right. \text { and } \\
& \left.F_{A g}(s[1, n]) \cap Q=\{s[\text { end }]\} \text { for some } Q \in \hbar(s[n], \Omega-A g)\right\} .
\end{aligned}
$$

Proof Immediately. 
2.2 Alternating bisimilarity and alternating approximate bisimilarity

To capture the behavioral equivalence between alternating transition systems associated with the same observation set and agent set, Alur et al. introduce the notion of alternating bisimilarity [3].

Definition 4 Let $T_{i}=\left(S_{i}, \mathbb{P}, \Omega, \Pi_{i}, \hbar_{i}\right)$ be two alternating transition systems $(i=1,2)$ and $A g \subseteq \Omega$. The binary relation $R \subseteq S_{1} \times S_{2}$ is said to be an $A g$-alternating bisimulation if and only if for any $\left(q_{1}, q_{2}\right) \in R$,

(1) $\Pi_{1}\left(q_{1}\right)=\Pi_{2}\left(q_{2}\right)$;

(2) $\forall Q_{1} \in \hbar_{1}\left(q_{1}, A g\right) \exists Q_{2} \in \hbar_{2}\left(q_{2}, A g\right) \forall Q_{2}^{\prime} \in \hbar_{2}\left(q_{2}, \Omega-A g\right) \exists Q_{1}^{\prime} \in \hbar_{1}\left(q_{1}, \Omega-\right.$ $A g)\left(Q_{1} \cap Q_{1}^{\prime}\right) \times\left(Q_{2} \cap Q_{2}^{\prime}\right) \subseteq R$;

(3) $\forall Q_{2} \in \hbar_{2}\left(q_{2}, A g\right) \exists Q_{1} \in \hbar_{1}\left(q_{1}, A g\right) \forall Q_{1}^{\prime} \in \hbar_{1}\left(q_{1}, \Omega-A g\right) \exists Q_{2}^{\prime} \in \hbar_{2}\left(q_{2}, \Omega-\right.$ $A g)\left(Q_{1} \cap Q_{1}^{\prime}\right) \times\left(Q_{2} \cap Q_{2}^{\prime}\right) \subseteq R$.

For any $q_{1} \in S_{1}$ and $q_{2} \in S_{2}$, these two states are said to be $A g$-alternating bisimilar, in symbols $q_{1} \sim_{A g} q_{2}$, if and only if there exists an $A g$-alternating bisimulation $R$ such that $\left(q_{1}, q_{2}\right) \in R$. In other words, $\sim_{A g} \triangleq \bigcup\left\{R \subseteq S_{1} \times S_{2}\right.$ : $R$ is an Ag-alternating bisimulation $\}$.

It is easy to check that $\sim_{A g}$ is an equivalence relation and is the largest $A g$-alternating bisimulation. We leave it to the interested readers. Alur et al. establish a modal characterization of alternating bisimilarity in terms of alternating-time temporal logic (for short, ATL) 3. They show that two states are $A g$-alternating bisimilar if and only if they satisfy the same $A g$-ATL formulas, where $A g$-ATL formulas are ATL-formulas in which all path quantifiers occurring are parameterized by $A g$.

Recently, alternating transition systems associated with metric over observations are adopted as models of the samples of control systems with disturbances and their finite abstractions 25] 26. In these work, the notion of alternating approximate bisimilarity is used to capture approximate equivalence between systems.

Definition 5 [26] Let $T_{i}=\left(S_{i}, \mathbb{P}, \Omega, \Pi_{i}, \hbar_{i}\right)$ be two alternating transition systems $(i=1,2)$ and $A g \subseteq \Omega$. Suppose that $d$ is a metric over $\mathbb{P}$ and $\varepsilon \in$ $\mathbb{R}_{+}^{0}$. The binary relation $R \subseteq S_{1} \times S_{2}$ is said to be an $(A g, \varepsilon)$-alternating approximate bisimulation if and only if for any $\left(q_{1}, q_{2}\right) \in R$,

(1) $d\left(\Pi_{1}\left(q_{1}\right), \Pi_{2}\left(q_{2}\right)\right) \leq \varepsilon$

(2) $\forall Q_{1} \in \hbar_{1}\left(q_{1}, A g\right) \exists Q_{2} \in \hbar_{2}\left(q_{2}, A g\right) \forall Q_{2}^{\prime} \in \hbar_{2}\left(q_{2}, \Omega-A g\right) \exists Q_{1}^{\prime} \in \hbar_{1}\left(q_{1}, \Omega-\right.$ $A g)\left(Q_{1} \cap Q_{1}^{\prime}\right) \times\left(Q_{2} \cap Q_{2}^{\prime}\right) \subseteq R$;

(3) $\forall Q_{2} \in \hbar_{2}\left(q_{2}, A g\right) \exists Q_{1} \in \hbar_{1}\left(q_{1}, A g\right) \forall Q_{1}^{\prime} \in \hbar_{1}\left(q_{1}, \Omega-A g\right) \exists Q_{2}^{\prime} \in \hbar_{2}\left(q_{2}, \Omega-\right.$ $A g)\left(Q_{1} \cap Q_{1}^{\prime}\right) \times\left(Q_{2} \cap Q_{2}^{\prime}\right) \subseteq R$.

Two states $q_{1} \in S_{1}$ and $q_{2} \in S_{2}$ are said to be $(A g, \varepsilon)$-alternating approximate bisimilar, denoted by $q_{1} \sim_{A g}^{\varepsilon} q_{2}$, if and only if there exists an $(A g, \varepsilon)$ alternating approximate bisimulation $R \subseteq S_{1} \times S_{2}$ such that $\left(q_{1}, q_{2}\right) \in R$.

\footnotetext{
${ }^{1}$ By Definition 1 it is easy to see that both $Q_{1} \cap Q_{1}^{\prime}$ and $Q_{2} \cap Q_{2}^{\prime}$ are singleton. Therefore, $\left(Q_{1} \cap Q_{1}^{\prime}\right) \times\left(Q_{2} \cap Q_{2}^{\prime}\right)$ is single.
} 
$T_{1}$ and $T_{2}$ are said to be $(A g, \varepsilon)$-alternating approximate bisimilar, in symbols $T_{1} \sim_{A g}^{\varepsilon} T_{2}$, if and only if $\left\{q_{1} \in S_{1}: q_{1} \sim_{A g}^{\varepsilon} q_{2}\right.$ for some $\left.q_{2} \in S_{2}\right\}=S_{1}$ and $\left\{q_{2} \in S_{2}: q_{1} \sim_{A g}^{\varepsilon} q_{2}\right.$ for some $\left.q_{1} \in S_{1}\right\}=S_{2}$.

The following results reveal some simple properties of $(A g, \varepsilon)$-alternating approximate bisimilarity.

Lemma 3 (1) $\sim_{A g}^{0}=\sim_{A g}$ and $\sim_{A g}^{0}$ is an equivalence relation;

(2) for any $\varepsilon_{1}, \varepsilon_{2} \in \mathbb{R}_{+}^{0}$, if $\varepsilon_{1} \leq \varepsilon_{2}$ then $\sim_{A g}^{\varepsilon_{1}} \subseteq \sim_{A g}^{\varepsilon_{2}}$;

(3) for any $\varepsilon \in \mathbb{R}_{+}^{0}, \sim_{A g}^{\varepsilon}$ is the largest $(A g, \varepsilon)$-alternating approximate bisimulation.

Proof (1) Since $d$ is a metric, we have $d\left(\Pi_{1}\left(q_{1}\right), \Pi_{2}\left(q_{2}\right)\right) \leq 0$ if and only if $\Pi_{1}\left(q_{1}\right)=\Pi_{2}\left(q_{2}\right)$ for any states $q_{1}$ and $q_{2}$. Thus it follows from Definition 4 and 5 that (1) holds.

(2) Immediately follows from Definition 5 .

(3) Let $\varepsilon \in \mathbb{R}_{+}^{0}$. According to Definition 5] it is not difficult to check that $(A g, \varepsilon)$-alternating approximate bisimulations are preserved under union. Thus $\sim_{A g}^{\varepsilon}$ is the largest $(A g, \varepsilon)$-alternating bisimulation.

As usual, $(A g, \varepsilon)$-alternating approximate bisimilarity can be characterized in the forth-back style. Formally, we have

Theorem $1 q_{1} \sim_{A g}^{\varepsilon} q_{2}$ if and only if the following hold:

(1) $d\left(\Pi_{1}\left(q_{1}\right), \Pi_{2}\left(q_{2}\right)\right) \leq \varepsilon$;

(2) $\forall Q_{1} \in \hbar_{1}\left(q_{1}, A g\right) \exists Q_{2} \in \hbar_{2}\left(q_{2}, A g\right) \forall Q_{2}^{\prime} \in \hbar_{2}\left(q_{2}, \Omega-A g\right) \exists Q_{1}^{\prime} \in \hbar_{1}\left(q_{1}, \Omega-\right.$ $A g)\left(\left(Q_{1} \cap Q_{1}^{\prime}\right) \times\left(Q_{2} \cap Q_{2}^{\prime}\right) \subseteq \sim_{A g}^{\varepsilon}\right)$;

(3) $\forall Q_{2} \in \hbar_{2}\left(q_{2}, A g\right) \exists Q_{1} \in \hbar_{1}\left(q_{1}, A g\right) \forall Q_{1}^{\prime} \in \hbar_{1}\left(q_{1}, \Omega-A g\right) \exists Q_{2}^{\prime} \in \hbar_{2}\left(q_{2}, \Omega-\right.$ $A g)\left(\left(Q_{1} \cap Q_{1}^{\prime}\right) \times\left(Q_{2} \cap Q_{2}^{\prime}\right) \subseteq \sim_{A g}^{\varepsilon}\right)$.

Proof (From left to right) Follows from Definition 5 and (3) in Lemma 3 .

(From right to left) Let $R \triangleq\left\{\left(q_{1}, q_{2}\right): q_{1}\right.$ and $q_{2}$ satisfy (1)-(3) $\} \cup \sim_{A g}^{\varepsilon}$. It is almost immediate to check that $R$ is an $(A g, \varepsilon)$-alternating approximate bisimulation. So by (3) in Lemma 3 , the conclusion holds.

It should be pointed out that $(A g, \varepsilon)$-alternating approximate bisimilarity is not always transitive and then is not always an equivalence relation. An example is given below.

Example 1 Consider the alternating transition system $\left(\left\{q_{1}, q_{2}, q_{3}\right\},\left\{p_{1}, p_{2}, p_{3}\right\}\right.$, $\{1\}, \Pi, \hbar)$, where $\Pi\left(q_{i}\right)=p_{i}$ and $\hbar\left(q_{i}, 1\right)=\left\{\left\{q_{i}\right\}\right\}$ for $i=1,2,3$. Let $A g=\{1\}$. Define a distance function $d$ over $\left\{p_{1}, p_{2}, p_{3}\right\}$ as: for any $p_{i}, p_{j} \in\left\{p_{1}, p_{2}, p_{3}\right\}$, $d\left(p_{i}, p_{j}\right)=|i-j|$. Clearly, this function $d$ is a metric. According to Definition 5 , it is not difficult to see that $p_{1} \sim_{A g}^{1} p_{2}, p_{2} \sim_{A g}^{1} p_{3}$ and $p_{1} \chi_{A g}^{1} p_{3}$. Thus $\sim_{A g}^{1}$ is not an equivalence relation. 


\section{$3 \mathrm{ATL}_{\varepsilon}, H_{A g}^{\varepsilon}$ and $E_{A g}^{\varepsilon}$}

In this and the next sections, we will establish a logical characterization of $(A g, \varepsilon)$-alternating approximate bisimilarity. To this end, a modal language is introduced below, which is obtained by adding the diamond operator $\langle\varepsilon\rangle$ to ATL.

Definition 6 Let $\varepsilon \in \mathbb{R}_{+}^{0}, \mathbb{P}$ a finite set of propositions and let $\Omega$ be a set of agents. $\mathrm{ATL}_{\varepsilon}(\mathbb{P}, \Omega)$ formulae are divided into: state formulas and path formulas, which are defined inductively as:

$$
\text { state formula } \varphi::=p|\langle\varepsilon\rangle p| \neg \varphi|\varphi \wedge \varphi|\langle\langle A g\rangle\rangle \phi,
$$

where $p \in \mathbb{P}, A g \subseteq \Omega$ and $\phi$ is a path formula;

$$
\text { path formula } \phi::=\varphi|\neg \phi| \phi \wedge \phi|X \phi| \phi \mathbf{U} \phi \text {, }
$$

where $\varphi$ is a state formula.

The operator $\left\langle\langle\rangle\right.$ is a path quantifier. Given $A g \subseteq \Omega$, an $\operatorname{ATL}_{\varepsilon}(\mathbb{P}, \Omega)$ formula $\alpha$ is said to be an $A g-\mathrm{ATL}_{\varepsilon}(\mathbb{P}, \Omega)$ formula if and only if all path quantifiers occurring in $\alpha$ are parameterized by $A g$.

As usual, logical connective $\vee$ can be defined in terms of $\neg$ and $\wedge$. If $\mathbb{P}$ and $\Omega$ are clear from the context, $\operatorname{ATL}_{\varepsilon}(\mathbb{P}, \Omega)$ and $A g-\mathrm{ATL}_{\varepsilon}(\mathbb{P}, \Omega)$ are often abbreviated to $\mathrm{ATL}_{\varepsilon}$ and $A g$-ATL $\mathrm{AT}_{\varepsilon}$, respectively. Henceforth, we use $\varphi, \gamma, \varphi_{1}, \gamma_{1} \ldots$ to denote state formulas and $\phi, \psi, \phi_{1}, \psi_{1}, \cdots$ to denote path formulas.

Definition 7 Let $T=(S, \mathbb{P}, \Omega, \Pi, \hbar)$ be an alternating transition system, $d$ a metric over $\mathbb{P}$ and $\varepsilon \in \mathbb{R}_{0}^{+}$. The satisfaction relation $\models_{s}\left(\models_{p}\right)$ between the states (the infinite state sequence $\sigma \in S^{\omega}$, respectively) of $T$ and state formulas (path formulas, respectively) is inductively defined as: for any $q \in S$ and $\sigma \in S^{\omega}$,

- $(T, d), q \models_{s} p$ iff $p=\Pi(q)$ for any $p \in \mathbb{P}$;

- $(T, d), q \models_{s}\langle\varepsilon\rangle p$ iff $d(p, \Pi(q)) \leq \varepsilon$;

- $(T, d), q \models_{s} \neg \varphi$ iff $T, q \models_{s} \varphi$ does not hold;

- $(T, d), q \models_{s} \varphi_{1} \wedge \varphi_{2}$ iff $(T, d), q \models_{s} \varphi_{1}$ and $(T, d), q \models_{s} \varphi_{2}$;

- $(T, d), q \models_{s}\langle\langle A g\rangle\rangle \phi$ iff there exists a strategy $F_{A g}$ of $A g$ such that $(T, d), \sigma \models_{p} \phi$ for any $\sigma \in O u t\left(q, F_{A g}\right)$;

- for any state formula $\varphi,(T, d), \sigma \models_{p} \varphi$ iff $(T, d), \sigma[1] \models_{s} \varphi$;

- $(T, d), \sigma \models_{p} \mathbf{X} \phi$ iff $(T, d), \sigma[2, \infty] \models_{p} \phi$;

- $(T, d), \sigma \models_{p} \phi_{1} \mathbf{U} \phi_{2}$ iff there exists $i \in \mathbb{N}$ such that $(T, d), \sigma[i, \infty] \models{ }_{p} \phi_{2}$ and for any $j<i,(T, d), \sigma[j, \infty] \models{ }_{p} \phi_{1}$;

- $(T, d), \sigma \models_{p} \neg \phi$ (or $\left.\phi_{1} \wedge \phi_{2}\right)$ can be defined similarly to $\models_{s}$.

For convenience, the subscripts of $\models_{s}$ and $\models_{p}$ will be omitted in this paper. In the following, two rank functions are introduced as usual.

Definition 8 Let $\varepsilon \in \mathbb{R}_{0}^{+}, \mathbb{P}$ a finite set of propositions and let $\Omega$ be a set of agents. The rank function $\xi_{s}\left(\xi_{p}\right)$ mapping $\mathrm{ATL}_{\varepsilon}$ state formulas (path formulas, respectively) to natural numbers is defined as: 
(1) for any $p \in \mathbb{P}, \xi_{s}(p)=1$ and $\xi_{s}(\langle\varepsilon\rangle p)=1$;

(2) $\xi_{s}(\neg \varphi)=\xi_{s}(\varphi)+1$;

(3) $\xi_{s}(\langle\langle A g\rangle\rangle \phi)=\xi_{p}(\phi)+1$;

(4) $\xi_{s}\left(\varphi_{1} \wedge \varphi_{2}\right)=\max \left\{\xi_{s}\left(\varphi_{1}\right), \xi_{s}\left(\varphi_{2}\right)\right\}+1$;

(5) for any state formula $\varphi, \xi_{p}(\varphi)=\xi_{s}(\varphi)+1$;

(6) $\xi_{p}(\neg \phi)=\xi_{p}(\phi)+1$;

(7) $\xi_{p}(\mathbf{X} \phi)=\xi_{p}(\phi)+1$;

(8) $\xi_{p}\left(\phi_{1} \wedge \phi_{2}\right)=\max \left\{\xi_{p}\left(\phi_{1}\right), \xi_{p}\left(\phi_{2}\right)\right\}+1$;

(9) $\xi_{p}\left(\phi_{1} \mathbf{U} \phi_{2}\right)=\max \left\{\xi_{p}\left(\phi_{1}\right), \xi_{p}\left(\phi_{2}\right)\right\}+1$.

This paper aims to establish a modal characterization of $(A g, \varepsilon)$-alternating approximate bisimilarity in terms of $A g-\mathrm{ATL}_{\varepsilon}$. However, as shown in Example1 $(A g, \varepsilon)$-alternating approximate bisimilarity is not always an equivalence relation. Then it may not coincide with modal equivalence w.r.t any modal logic. In other words, the modal characterization of $(A g, \varepsilon)$-alternating approximate bisimilarity can not be provided in the usual style.

To overcome this defect, two binary relations between formulas will be introduced, which will play the central roles in this paper. Before giving them formally, we explain the motivation behind these notions. Recall that two states are $(A g, \varepsilon)$-alternating approximate bisimilar if and only if they satisfy the forth and back conditions in Theorem 1. So, in order to establish the modal characterization of $(A g, \varepsilon)$-alternating approximate bisimilarity, we need to formalize these conditions in terms of $\mathrm{ATL}_{\varepsilon}$ formulas. According to the semantics of $\mathrm{ATL}_{\varepsilon}$, we have the following observation.

For any $\varepsilon \in \mathbb{R}_{0}^{+}$, state $q_{1}$ of $T_{1}$ and state $q_{2}$ of $T_{2}, q_{1} \sim_{A g}^{\varepsilon} q_{2}$ implies that for each $p \in \mathbb{P},\left(T_{1}, d\right), q_{1} \models p$ implies $\left(T_{2}, d\right), q_{2} \models\langle\varepsilon\rangle p$ and vice verse.

This simple observation gives us a hint about the logical characterization of $(A g, \varepsilon)$-alternating approximate bisimilarity. That is, we may characterize it in terms of an appropriate binary relation $H$ over $\mathrm{ATL}_{\varepsilon}$ state formulae, and this characterization will possess the form " $q_{1} \sim_{A g}^{\varepsilon} q_{2}$ iff for any pair $(\varphi$, $\gamma) \in H,\left(T_{1}, d\right), q_{1} \models \varphi$ implies $\left(T_{2}, d\right), q_{2} \models \gamma$, and vice versa". To provide such relation $H$, we introduce the notions below.

Definition 9 Let $\mathbb{P}$ be a finite set of propositions, $\varepsilon \in \mathbb{R}_{+}^{0}, \Omega$ a set of agents

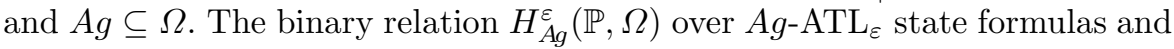
the binary relation $E_{A g}^{\varepsilon}(\mathbb{P}, \Omega)$ over $A g$-ATL $\mathrm{AT}_{\varepsilon}$ path formulas are the smallest pair of relations satisfying the following conditions (i.e., for any pair of relations $H$ and $E$ over states formulas and path formulas, respectively, if they satisfy the following conditions then $H_{A g}^{\varepsilon}(\mathbb{P}, \Omega) \subseteq H$ and $\left.E_{A g}^{\varepsilon}(\mathbb{P}, \Omega) \subseteq E\right)$ :

(1) for any $p \in \mathbb{P},(p,\langle\varepsilon\rangle p) \in H_{A g}^{\varepsilon}(\mathbb{P}, \Omega)$;

(2) if $(\varphi, \gamma) \in H_{A g}^{\varepsilon}(\mathbb{P}, \Omega)$, then $(\neg \gamma, \neg \varphi) \in H_{A g}^{\varepsilon}(\mathbb{P}, \Omega)$;

(3) if $\left(\varphi_{i}, \gamma_{i}\right) \in H_{A g}^{\varepsilon}(\mathbb{P}, \Omega)$ for $i=1,2$, then $\left(\varphi_{1} \wedge \varphi_{2}, \gamma_{1} \wedge \gamma_{2}\right) \in H_{A g}^{\varepsilon}(\mathbb{P}, \Omega)$;

(4) if $(\psi, \phi) \in E_{A g}^{\varepsilon}(\mathbb{P}, \Omega)$, then $(\langle\langle A g\rangle\rangle \psi,\langle\langle A g\rangle\rangle \phi) \in H_{A g}^{\varepsilon}(\mathbb{P}, \Omega)$;

(5) if $(\varphi, \gamma) \in H_{A g}^{\varepsilon}(\mathbb{P}, \Omega)$, then $(\varphi, \gamma) \in E_{A g}^{\varepsilon}(\mathbb{P}, \Omega)$;

(6) if $(\psi, \phi) \in E_{A g}^{\varepsilon}(\mathbb{P}, \Omega)$, then $(\neg \phi, \neg \psi) \in E_{A g}^{\varepsilon}(\mathbb{P}, \Omega)$;

(7) if $\left(\psi_{i}, \phi_{i}\right) \in E_{A g}^{\varepsilon}(\mathbb{P}, \Omega)$ for $i=1,2$, then $\left(\psi_{1} \wedge \psi_{2}, \phi_{1} \wedge \phi_{2}\right) \in E_{A g}^{\varepsilon}(\mathbb{P}, \Omega)$; 
(8) if $(\psi, \phi) \in E_{A g}^{\varepsilon}(\mathbb{P}, \Omega)$, then $(\mathbf{X} \psi, \mathbf{X} \phi) \in E_{A g}^{\varepsilon}(\mathbb{P}, \Omega)$;

(9) if $\left(\psi_{i}, \phi_{i}\right) \in E_{A g}^{\varepsilon}(\mathbb{P}, \Omega)$ for $i=1,2$, then $\left(\psi_{1} \mathbf{U} \psi_{2}, \phi_{1} \mathbf{U} \phi_{2}\right) \in E_{A g}^{\varepsilon}(\mathbb{P}, \Omega)$.

For convenience, if $\mathbb{P}$ and $\Omega$ are clear from the context, $H_{A g}^{\varepsilon}(\mathbb{P}, \Omega)$ and $E_{A g}^{\varepsilon}(\mathbb{P}, \Omega)$ are often abbreviated to $H_{A g}^{\varepsilon}$ and $E_{A g}^{\varepsilon}$, respectively. The following result guarantees the existence of these two relations.

Proposition 1 Let $\mathbb{P}$ be a finite set of propositions, $\varepsilon \in \mathbb{R}_{+}^{0}, \Omega$ a set of agents and $A g \subseteq \Omega$. Then

(i) Let $I$ be an index set. If for each $i \in I$, the binary relation $H_{i}$ over $A g$ $A T L_{\varepsilon}$ state formulas and the binary relation $E_{i}$ over $A g-A T L_{\varepsilon}$ path formulas satisfy the conditions in Definition [9, then so is $\left(\bigcap_{i \in I} H_{i}, \bigcap_{i \in I} E_{i}\right)$.

(ii) The smallest pair of relations satisfying the conditions in Definition 9 exist.

Proof Clearly, the pair of relations $H$ and $E$ satisfy the conditions in Definition 9, where $H \triangleq\left\{\left(\varphi_{1}, \varphi_{2}\right): \varphi_{1}\right.$ and $\varphi_{2}$ are $A g-\mathrm{ATL}_{\varepsilon}$ state formulas $\}$ and $E \triangleq\left\{\left(\phi_{1}, \phi_{2}\right): \phi_{1}\right.$ and $\phi_{2}$ are $A g-\mathrm{ATL}_{\varepsilon}$ path formulas $\}$. So it follows from (i) that (ii) holds. We prove (i) below.

Assume that for each $i \in I$, the binary relation $H_{i}$ over $A g-\mathrm{ATL}_{\varepsilon}$ state formulas and the binary relation $E_{i}$ over $A g-\mathrm{ATL}_{\varepsilon}$ path formulas satisfy the conditions in Definition 9. It suffices to show that the pair $\left(\bigcap_{i \in I} H_{i}, \bigcap_{i \in I} E_{i}\right)$ satisfies the conditions (1)-(9) in Definition 9. We will provide two sample cases.

(1) Let $p \in \mathbb{P}$. Then for each $i \in I$, since $H_{i}$ and $E_{i}$ satisfy the conditions in Definition 9] it follows that $(p,\langle\varepsilon\rangle p) \in H_{i}$. Thus we have $(p,\langle\varepsilon\rangle p) \in \bigcap_{i \in I} H_{i}$.

(2) Let $(\varphi, \gamma) \in \bigcap_{i \in I} H_{i}$. Then $(\varphi, \gamma) \in H_{i}$ for each $i \in I$. So for each $i \in I$, since $H_{i}$ and $E_{i}$ satisfy the conditions in Definition 9, we get $(\neg \gamma, \neg \varphi) \in H_{i}$. Therefore, we obtain $(\neg \gamma, \neg \varphi) \in \bigcap_{i \in I} H_{i}$

A few of useful properties of $H_{A g}^{\varepsilon}$ and $E_{A g}^{\varepsilon}$ are listed below.

Lemma 4 For any $(\varphi, \gamma) \in H_{A g}^{\varepsilon}$ and $(\psi, \phi) \in E_{A g}^{\varepsilon}$, the following hold:

(a) $\varphi$ is in one of the following forms: $p, \neg \gamma_{1}, \varphi_{1} \wedge \varphi_{2}$ and $\langle\langle A g\rangle\rangle \psi$;

(b) $\varphi$ and $\psi$ can not be in the form of $\langle\varepsilon\rangle p$;

(c) if $\varphi=p$, then $\gamma=\langle\varepsilon\rangle p$ and $\xi_{s}(\varphi)=\xi_{s}(\gamma)=1$;

(d) if $\varphi=\neg \gamma_{1}$, then there exists a state formula $\varphi_{1}$ such that $\left(\varphi_{1}, \gamma_{1}\right) \in$ $H_{A g}^{\varepsilon}, \gamma=\neg \varphi_{1}$ and $\xi_{s}(\varphi)=\xi_{s}(\gamma)=\xi_{s}\left(\gamma_{1}\right)+1$;

(e) if $\varphi=\varphi_{1} \wedge \varphi_{2}$, then there exist state formulas $\gamma_{1}$ and $\gamma_{2}$ such that $\left(\varphi_{i}, \gamma_{i}\right) \in H_{A g}^{\varepsilon}(i=1,2), \gamma=\gamma_{1} \wedge \gamma_{2}$ and $\xi_{s}(\varphi)=\xi_{s}(\gamma)=\max \left\{\xi_{s}\left(\gamma_{1}\right), \xi_{s}\left(\gamma_{2}\right)\right\}+$ 1 ;

(f) if $\varphi=\langle\langle A g\rangle\rangle \psi_{1}$, then there exists a path formula $\phi_{1}$ such that $\left(\psi_{1}, \phi_{1}\right) \in$ $E_{A g}^{\varepsilon}, \gamma=\langle\langle A g\rangle\rangle \phi_{1}$ and $\xi_{s}(\varphi)=\xi_{s}(\gamma)=\xi_{p}\left(\psi_{1}\right)+1$;

(g) if the path formula $\psi$ is also a state formula, then $\phi$ is also a state formula, $(\psi, \phi) \in H_{A g}^{\varepsilon}$ and $\xi_{p}(\phi)=\xi_{p}(\psi)=\xi_{s}(\psi)+1$;

(h) if $\psi=\neg \phi_{1}$, then there exists a path formula $\psi_{1}$ such that $\left(\psi_{1}, \phi_{1}\right) \in$ $E_{A g}^{\varepsilon}, \phi=\neg \psi_{1}$ and $\xi_{p}(\phi)=\xi_{p}(\psi)=\xi_{p}\left(\psi_{1}\right)+1$; 
(i) if $\psi=\psi_{1} \wedge \psi_{2}$, then there exist path formulas $\phi_{1}$ and $\phi_{2}$ such that $\left(\psi_{i}, \phi_{i}\right) \in E_{A g}^{\varepsilon}(i=1,2), \phi=\phi_{1} \wedge \phi_{2}$ and $\xi_{p}(\phi)=\xi_{p}(\psi)=\max \left\{\xi_{p}\left(\psi_{1}\right), \xi_{p}\left(\psi_{2}\right)\right\}+$ 1 ;

(j) if $\psi=\mathbf{X} \psi_{1}$, then there exists a path formula $\phi_{1}$ such that $\left(\psi_{1}, \phi_{1}\right) \in$ $E_{A g}^{\varepsilon}, \phi=\mathbf{X} \phi_{1}$ and $\xi_{p}(\phi)=\xi_{p}(\psi)=\xi_{p}\left(\psi_{1}\right)+1$;

$(k)$ if $\psi=\psi_{1} \mathbf{U} \psi_{2}$, then there exist path formulas $\phi_{1}$ and $\phi_{2}$ such that $\left(\psi_{i}, \phi_{i}\right) \in E_{A g}^{\varepsilon}(i=1,2), \phi=\phi_{1} \mathbf{U} \phi_{2}$ and $\xi_{p}(\phi)=\xi_{p}(\psi)=\max \left\{\xi_{p}\left(\psi_{1}\right), \xi_{p}\left(\psi_{2}\right)\right\}+$ 1 .

Proof Straightforward.

It follows from the above result that $(p,\langle\varepsilon\rangle p) \in H_{A g}^{\varepsilon}$ and $(p,\langle\varepsilon\rangle p) \in E_{A g}^{\varepsilon}$ but neither $(\langle\varepsilon\rangle p, p) \in H_{A g}^{\varepsilon}$ nor $(\langle\varepsilon\rangle p, p) \in E_{A g}^{\varepsilon}$. Thus neither $H_{A g}^{\varepsilon}$ nor $E_{A g}^{\varepsilon}$ is symmetric and then none of them is an equivalence relation. For $\varepsilon=0$, some internal relations between $H_{A g}^{\varepsilon}$ (or, $\left.E_{A g}^{\varepsilon}\right)$ and $\models_{s}\left(\models_{p}\right.$, respectively) are revealed in the next lemma.

Lemma 5 Let $T=(S, \mathbb{P}, \Omega, \Pi, \hbar)$ be an alternating transition system and let $d$ be a metric over $\mathbb{P}$. Then

(1) for any $q \in S$ and $(\varphi, \gamma) \in H_{A g}^{0},(T, d), q \models \varphi$ if and only if $(T, d), q \models$

(2) for any $\sigma \in S^{\omega}$ and $(\psi, \phi) \in E_{A g}^{0},(T, d), \sigma \models \psi$ if and only if $(T, d), \sigma \models$ $\phi$;

(3)for any $A g-A T L_{0}$ state formula $\varphi_{0}$, there exists $(\varphi, \gamma) \in H_{A g}^{0}$ such that for any $q \in S,(T, d), q \models \varphi_{0}$ if and only if $(T, d), q \models \varphi$;

(4) for any $A g-A T L_{0}$ path formula $\psi_{0}$, there exists $(\psi, \phi) \in E_{A g}^{0}$ such that for any $\sigma \in S^{\omega},(T, d), \sigma \models \psi_{0}$ if and only if $(T, d), \sigma \models \psi$.

Proof Since $d$ is a metric over $\mathbb{P}$, for any $p, p^{\prime} \in \mathbb{P}, d\left(p, p^{\prime}\right) \leq 0$ if and only if $p=p^{\prime}$. Further, by Definition 7 and Lemma 4, it is easy to prove (1) and (2) by induction on the ranks of $\varphi$ and $\psi$. Next, we prove (3) and (4) simultaneously by induction on the ranks of $\varphi_{0}$ and $\psi_{0}$.

By Definition 8, it is clear that if $\xi_{s}\left(\varphi_{0}\right)=0$ and $\xi_{p}\left(\psi_{0}\right)=0$ then $(3)$ and (4) hold.

Suppose that $\xi_{s}\left(\varphi_{0}\right)=\xi_{p}\left(\psi_{0}\right)=n+1$ and the items (3) and (4) hold for any $A g-\mathrm{ATL}_{0}$ state formula $\varphi$ and $A g-\mathrm{ATL}_{0}$ path formula $\psi$ with $\xi_{s}(\varphi) \leq n$ and $\xi_{p}(\psi) \leq n$. According to Definition $8, \varphi_{0}$ is in one of the following forms: $p,\langle 0\rangle p, \neg \gamma_{1}, \varphi_{1} \wedge \varphi_{2}$ and $\langle\langle A g\rangle\rangle \psi$, and $\psi_{0}$ is in the form of $\varphi, \neg \phi_{1}, \psi_{1} \wedge \psi_{2}, \mathbf{X} \psi$ or $\psi_{1} \mathbf{U} \psi_{2}$. In the following, we just provide two sample cases. The proofs of other cases are similar.

Suppose that $\varphi_{0}=\langle 0\rangle p$ for some $p \in \mathbb{P}$. It follows from (1) and $(p,\langle 0\rangle p) \in$ $H_{A q}^{0}$ that for any $q \in S,(T, d), q \models \varphi_{0}$ if and only if $(T, d), q \models p$. We set $\varphi \triangleq p$ and $\gamma \triangleq\langle 0\rangle p$. Clearly, $(\varphi, \gamma) \in H_{A g}^{0}$ and for any $q \in S,(T, d), q \models \varphi_{0}$ if and only if $(T, d), q \models \varphi$.

Suppose that $\varphi_{0}=\langle\langle A g\rangle\rangle \psi$. Then by Definition 8, we get $\xi_{p}(\psi)=n$. Further, by induction hypothesis, there exists $\left(\psi^{\prime}, \phi^{\prime}\right) \in E_{A g}^{0}$ such that for any $\sigma \in S^{\omega},(T, d), \sigma \models \psi$ if and only if $(T, d), \sigma \models \psi^{\prime}$. We set $\varphi \triangleq\langle\langle A g\rangle\rangle \psi^{\prime}$ and 
$\gamma \triangleq\langle\langle A g\rangle\rangle \phi^{\prime}$. Then it follows from Definition 9 that $(\varphi, \gamma) \in H_{A g}^{0}$. Moreover, by Definition 7 it is clear that for any $q \in S,(T, d), q \models \varphi_{0}$ if and only if $(T, d), q \models \varphi$.

Proposition 2 Let $T=(S, \mathbb{P}, \Omega, \Pi, \hbar)$ be an alternating transition system, $d$ a metric over $\mathbb{P}$ and $\varepsilon \in \mathbb{R}_{+}^{0}$. Then for any $q \in S, \sigma \in S^{\omega},(\varphi, \gamma) \in H_{\text {Ag }}^{\varepsilon}$ and $(\psi, \phi) \in E_{A g}^{\varepsilon}$, the following conclusions hold:

(1) if $(T, d), q \models \varphi$ then $(T, d), q \models \gamma$;

(2) if $(T, d), \sigma \models \psi$ then $(T, d), \sigma \models \phi$.

Proof We prove (1) and (2) simultaneously by induction on the ranks of $\varphi$ and $\psi$.

By Definition 8 if $\xi_{s}(\varphi)=0$ and $\xi_{p}(\psi)=0$ then the conclusions hold trivially.

Suppose that $\xi_{s}(\varphi)=\xi_{p}(\psi)=n+1,(\varphi, \gamma) \in H_{A g}^{\varepsilon},(\psi, \phi) \in E_{A g}^{\varepsilon}$ and the items (1) and (2) hold for any $\left(\varphi_{0}, \gamma_{0}\right) \in H_{A g}^{\varepsilon}$ and $\left(\psi_{0}, \phi_{0}\right) \in E_{A g}^{\varepsilon}$ with $\xi_{s}\left(\varphi_{0}\right) \leq n$ and $\xi_{p}\left(\psi_{0}\right) \leq n$. By Definition 6 , $\varphi$ is in one of the following forms: $p, \neg \gamma_{1}, \varphi_{1} \wedge \varphi_{2}$ and $\langle\langle A g\rangle\rangle \psi_{1}$, and $\psi$ is in the form of $\varphi_{1}, \neg \phi_{1}, \psi_{1} \wedge \psi_{2}, \mathbf{X} \psi_{1}$ or $\psi_{1} \mathbf{U} \psi_{2}$. In the following, we just provide some sample cases.

Case $1 \varphi=p$ for some $p \in \mathbb{P}$. Then by Lemma 4, we obtain $\gamma=\langle\varepsilon\rangle p$. Let $q \in S$ and $(T, d), q \models \varphi$. Then it follows from Definition 7 that $\Pi(q)=p$. Since $d$ is a metric, we have $d(p, \Pi(q))=d(p, p)=0 \leq \varepsilon$. Thus by Definition 7 , we get $(T, d), q \models\langle\varepsilon\rangle p$.

Case $2 \varphi=\langle\langle A g\rangle\rangle \psi_{0}$. Let $q \in S$ and $(T, d), q \models \varphi$. Due to Lemma 4 there exists a path formula $\phi_{0}$ such that $\left(\psi_{0}, \phi_{0}\right) \in E_{A g}^{\varepsilon}, \gamma=\langle\langle A g\rangle\rangle \phi_{0}$ and $\xi_{s}(\varphi)=\xi_{s}(\gamma)=\xi_{p}\left(\psi_{0}\right)+1$. By Definition 7 and $(T, d), q \models \varphi$, there is a strategy $F_{A g}$ of $A g$ such that $(T, d), \sigma \models \psi_{0}$ for any $\sigma \in O u t\left(q, F_{A g}\right)$. Then by induction hypothesis, $(T, d), \sigma \models \phi_{0}$ for any $\sigma \in \operatorname{Out}\left(q, F_{A g}\right)$. Therefore, by Definition 7 we have $(T, d), q \models \gamma$.

Case $3 \psi=\psi_{1} \mathbf{U} \psi_{2}$. Let $\sigma \in S^{\omega}$ and $(T, d), \sigma \models \psi$. It follows from Lemma 4 that for some path formulas $\phi_{1}$ and $\phi_{2},\left(\psi_{i}, \phi_{i}\right) \in E_{A g}^{\varepsilon}(i=1,2), \phi=\phi_{1} \mathbf{U} \phi_{2}$ and $\xi_{p}(\psi)=\xi_{p}(\phi)=\max \left\{\xi_{p}\left(\psi_{1}\right), \xi_{p}\left(\psi_{2}\right)\right\}+1$. Since $(T, d), \sigma \models \psi$, by Definition 7 there exists $i \in \mathbb{N}$ such that $(T, d), \sigma[i, \infty] \models \psi_{2}$ and $(T, d), \sigma[j, \infty] \models$ $\psi_{1}$ for any $j<i$. Then by induction hypothesis, $(T, d), \sigma[i, \infty] \models \phi_{2}$ and $(T, d), \sigma[j, \infty] \models \phi_{1}$ for any $j<i$. Therefore, by Definition 7 , we obtain $(T, d), \sigma \models \phi$.

\section{Modal characterization of alternating approximate bisimilarity}

This section will establish a modal characterization of $(A g, \varepsilon)$-alternating approximate bisimilarity in terms of relations $H_{A g}^{\varepsilon}$ and $E_{A g}^{\varepsilon}$ defined in the previous section. Similar method has been adopted to provide the modal characterization of $\lambda$-bisimilarity [35. In order to obtain such modal characterization, a number of auxiliary lemmas are needed.

Firstly, we intend to demonstrate that for any alternating approximately bisimilar states $q_{1}$ (of $T_{1}$ ) and $q_{2}$ (of $T_{2}$ ), given a strategy of $T_{1}$, there exists a 
strategy of $T_{2}$ such that, under control of these strategies, each trace starting from $q_{2}$ is approximately bisimilar to some trace starting from $q_{1}$. To prove this conclusion, we need könig's lemma (see [19]), which says

every infinite, finite branching tree has an infinite branch.

Lemma 6 Let $T_{i}=\left(S_{i}, \mathbb{P}, \Omega, \Pi_{i}, \hbar_{i}\right)$ be two finite branching alternating transition systems $(i=1,2)$. Suppose that $d$ is a metric over $\mathbb{P}, \varepsilon \in \mathbb{R}_{+}^{0}, A g \subseteq \Omega$ and $F_{A g}:\left(S_{1}\right)^{+} \rightarrow 2^{S_{1}}$ is a strategy of Ag. For any $q_{1} \in S_{1}$ and $q_{2} \in S_{2}$ with $q_{1} \sim_{A g}^{\varepsilon} q_{2}$, there is a strategy $F_{A g}^{\prime}:\left(S_{2}\right)^{+} \rightarrow 2^{S_{2}}$ such that for any $\sigma_{2} \in \operatorname{Out}\left(q_{2}, F_{A g}^{\prime}\right), \sigma_{1} \sim_{A g}^{\varepsilon} \sigma_{2}$ for some $\sigma_{1} \in \operatorname{Out}\left(q_{1}, F_{A g}\right) 2$.

Proof Let $q_{1} \in S_{1}, q_{2} \in S_{2}$ and $q_{1} \sim_{A g}^{\varepsilon} q_{2}$. To obtain the desired strategy $F_{A g}^{\prime}:\left(S_{2}\right)^{+} \rightarrow 2^{S_{2}}$, we define subsets $\Delta_{n}$ of $\left(S_{2}\right)^{n}$ and functions $F_{n}: \Delta_{n} \rightarrow$ $2^{S_{2}}(n \in \mathbb{N})$ by induction on $n$ as follows.

We set $\Delta_{1} \triangleq\left\{q_{2}\right\}$. Since $F_{A g}$ is a strategy of $A g$, we get $F_{A g}\left(q_{1}\right) \in$ $\hbar_{1}\left(q_{1}, A g\right)$. Then by $q_{1} \sim_{A g}^{\varepsilon} q_{2}$ and Theorem 11, there exists $Q_{2} \in \hbar_{2}\left(q_{2}, A g\right)$ such that for any $Q_{2}^{\prime} \in \hbar_{2}\left(q_{2}, \Omega-A g\right),\left(Q_{1}^{\prime} \cap F_{A g}\left(q_{1}\right)\right) \times\left(Q_{2} \cap Q_{2}^{\prime}\right) \subseteq \sim_{A g}^{\varepsilon}$ for some $Q_{1}^{\prime} \in \hbar_{1}\left(q_{1}, \Omega-A g\right)$. Note that such $Q_{2}$ may not be unique. Choose and fix an arbitrary such $Q_{2}$ and set $F_{1}\left(q_{2}\right) \triangleq Q_{2}$. Clearly, $F_{1}$ is a function from $\Delta_{1}$ to $2^{S_{2}}$.

Suppose that $\Delta_{k}$ and $F_{k}$ have been defined. We define $\Delta_{k+1}$ and $F_{k+1}$ below. We set

$\Delta_{k+1} \triangleq\left\{s_{2} q_{2}^{\prime}: s_{2} \in \Delta_{k}\right.$ and $q_{2}^{\prime} \in F_{k}\left(s_{2}\right) \cap Q_{2}^{\prime}$ for some $Q_{2}^{\prime} \in \hbar_{2}\left(s_{2}[\right.$ end $\left.\left.], \Omega-A g\right)\right\}$.

For any $s_{2}^{\prime} \in \Delta_{k+1}$, if there does not exist $s_{1}^{\prime} \in O u t^{k+1}\left(q_{1}, F_{A g}\right)$ such that $s_{1}^{\prime} \sim_{A g}^{\varepsilon} s_{2}^{\prime}$, then we set $F_{k+1}\left(s_{2}^{\prime}\right)=S_{2}$; if there exists $s_{1}^{\prime} \in O u t^{k+1}\left(q_{1}, F_{A g}\right)$ such that $s_{1}^{\prime} \sim_{A g}^{\varepsilon} s_{2}^{\prime}$, then by Theorem 1, we have

$$
\begin{aligned}
\exists Q_{2} \in \hbar_{2}\left(s_{2}^{\prime}[e n d], A g\right) & \left(\forall Q_{2}^{\prime} \in \hbar_{2}\left(s_{2}^{\prime}[e n d], \Omega-A g\right) \exists Q_{1}^{\prime} \in \hbar_{1}\left(s_{1}^{\prime}[e n d], \Omega-A g\right)\right. \\
& \left.\left(\left(Q_{1}^{\prime} \cap F_{A g}\left(s_{1}^{\prime}\right)\right) \times\left(Q_{2} \cap Q_{2}^{\prime}\right) \subseteq \sim_{A g}^{\varepsilon}\right)\right) .
\end{aligned}
$$

We choose such a $Q_{2}$ and set $F_{k+1}\left(s_{2}^{\prime}\right) \triangleq Q_{2}$. Clearly, $F_{k+1}$ is a function from $\Delta_{k+1}$ to $2^{S_{2}}$.

On the other hand, by Lemma 1, there exists at least one strategy of $\mathrm{Ag}$ mapping $\left(S_{2}\right)^{+}$to $2^{S_{2}}$. Let $F_{A g}^{\prime \prime}:\left(S_{2}\right)^{+} \rightarrow 2^{S_{2}}$ be an arbitrary strategy of $\mathrm{Ag}$. Define the function $F_{A g}^{\prime}:\left(S_{2}\right)^{+} \rightarrow 2^{S_{2}}$ as follows: for any $s \in\left(S_{2}\right)^{+}$,

$$
F_{A g}^{\prime}(s)= \begin{cases}F_{|s|}(s) & \text { if } s \in \Delta_{|s|} \\ F_{A g}^{\prime \prime}(s) & \text { otherwise }\end{cases}
$$

Next we want to show that the function $F_{A g}^{\prime}$ is the desired strategy. It is enough to prove three claims below.

\footnotetext{
${ }^{2} \sigma_{1} \sim \sim_{A g}^{\varepsilon} \sigma_{2}$ if and only if for any $i \in \mathbb{N}, \sigma_{1}[i] \sim_{A g}^{\varepsilon} \sigma_{2}[i]$. Similarly, $s_{1} \sim_{A g}^{\varepsilon} s_{2}$ if and only if $s_{1}[i] \sim_{A g}^{\varepsilon} s_{2}[i]$ for any $i \leq \max \left\{\left|s_{1}\right|,\left|s_{2}\right|\right\}$.
} 
Claim 1 For any $n \in \mathbb{N}$, the following conclusions hold:

$\left(1_{n}\right) \Delta_{n} \neq \emptyset$

$\left(2_{n}\right)$ for any $s_{2} \in \Delta_{n}, s_{1} \sim_{A g}^{\varepsilon} s_{2}$ for some $s_{1} \in O u t^{n}\left(q_{1}, F_{A g}\right)$;

$\left(3_{n}\right)$ for any $s_{2} \in \Delta_{n}, F_{n}\left(s_{2}\right) \in \hbar_{2}\left(s_{2}[\right.$ end $\left.], A g\right)$.

We proceed by induction on $n$.

If $n=1$, then $\left(1_{n}\right)$ and $\left(2_{n}\right)$ hold trivially. By the definition of $F_{1}$, we obtain $F_{1}\left(q_{2}\right) \in \hbar_{2}\left(q_{2}, A g\right)$. Thus $\left(3_{n}\right)$ holds due to $\Delta_{1}=\left\{q_{2}\right\}$.

Suppose that $\left(1_{k}\right),\left(2_{k}\right)$ and $\left(3_{k}\right)$ hold. We prove $\left(1_{k+1}\right),\left(2_{k+1}\right)$ and $\left(3_{k+1}\right)$ in turn.

$\left(1_{k+1}\right)$ By induction hypothesis, we get $\Delta_{k} \neq \emptyset$ and $F_{k}(s) \in \hbar_{2}(s[$ end $], A g)$ for any $s \in \Delta_{k}$. Let $s_{2} \in \Delta_{k}$ and $Q_{2}^{\prime} \in \hbar_{2}\left(s_{2}[e n d], \Omega-A g\right)$. By Definition 1 . $F_{k}\left(s_{2}\right) \cap Q_{2}^{\prime}$ is a singleton set. That is, there exists $q_{2}^{\prime} \in S_{2}$ such that $F_{k}\left(s_{2}\right) \cap$ $Q_{2}^{\prime}=\left\{q_{2}^{\prime}\right\}$. Therefore, it follows from the definition of $\Delta_{k+1}$ that $s_{2} q_{2}^{\prime} \in \Delta_{k+1}$. Thus $\Delta_{k+1} \neq \emptyset$.

$\left(2_{k+1}\right)$ Let $s_{2}^{\prime} \in \Delta_{k+1}$. By the construction of $\Delta_{k+1}$, there exists $s_{2} \in \Delta_{k}$, $Q_{2}^{\prime} \in \hbar\left(s_{2}[e n d], \Omega-A g\right)$ and $q_{2}^{\prime} \in F_{k}\left(s_{2}\right) \cap Q_{2}^{\prime}$ such that $s_{2}^{\prime}=s_{2} q_{2}^{\prime}$. By induction hypothesis, $s_{1} \sim_{A g}^{\varepsilon} s_{2}$ for some $s_{1} \in O u t^{k}\left(q_{1}, F_{A g}\right)$. Then by the construction of $F_{k}$, there exists $Q_{1}^{\prime} \in \hbar_{1}\left(s_{1}[e n d], \Omega-A g\right)$ such that

$$
\left(Q_{1}^{\prime} \cap F_{A g}\left(s_{1}\right)\right) \times\left(F_{k}\left(s_{2}\right) \cap Q_{2}^{\prime}\right) \subseteq \sim_{A g}^{\varepsilon} .
$$

According to Definition 1, both $Q_{1}^{\prime} \cap F_{A g}\left(s_{1}\right)$ and $F_{k}\left(s_{2}\right) \cap Q_{2}^{\prime}$ are singleton sets. Thus there exists $q_{1}^{\prime} \in S_{1}$ such that $Q_{1}^{\prime} \cap F_{A g}\left(s_{1}\right)=\left\{q_{1}^{\prime}\right\}$ and $q_{1}^{\prime} \sim_{A g}^{\varepsilon} q_{2}^{\prime}$. Then it follows that $s_{1} q_{1}^{\prime} \sim_{A g}^{\varepsilon} s_{2}^{\prime}$. Moreover, by Lemma 2] we have $s_{1} q_{1}^{\prime} \in$ Out ${ }^{k+1}\left(q_{1}, F_{A g}\right)$, as desired.

$\left(3_{k+1}\right)$ Let $s_{2}^{\prime} \in \Delta_{k+1}$. It follows from $\left(2_{k+1}\right)$ that $s_{1}^{\prime} \sim_{A g}^{\varepsilon} s_{2}^{\prime}$ for some $s_{1}^{\prime} \in \operatorname{Out}^{k+1}\left(q_{1}, F_{A g}\right)$. Then by the definition of $F_{k+1}$, we get $F_{k+1}\left(s_{2}^{\prime}\right) \in$ $\hbar_{2}\left(s_{2}^{\prime}[e n d], A g\right)$.

Claim $2 F_{A g}^{\prime}$ is a strategy of $A g$ and for any $n \in \mathbb{N}, \Delta_{n}=O u t^{n}\left(q_{2}, F_{A g}^{\prime}\right)$.

By Claim 1 and the definition of $F_{A g}^{\prime}, F_{A g}^{\prime}\left(s_{2}\right) \in \hbar_{2}\left(s_{2}[e n d], A g\right)$ for any $s_{2} \in\left(S_{2}\right)^{+}$. Thus by Lemma 1, $F_{A g}^{\prime}$ is a strategy of $A g$. Next we prove that for any $n \in \mathbb{N}, \Delta_{n}=\operatorname{Out}^{n}\left(q_{2}, F_{A g}^{\prime}\right)$.

If $n=1$ then $\Delta_{n}=\left\{q_{2}\right\}=\operatorname{Out}^{n}\left(q_{2}, F_{A g}^{\prime}\right)$ holds trivially.

Suppose that $n=k+1$ and $\Delta_{k}=O u t^{k}\left(q_{2}, F_{A g}^{\prime}\right)$. Then by the definition of $F_{A g}^{\prime}, F_{A g}^{\prime}\left(s_{2}\right)=F_{k}\left(s_{2}\right)$ for any $s_{2} \in \Delta_{k}$. Further, since $\Delta_{k}=O u t^{k}\left(q_{2}, F_{A g}^{\prime}\right)$, by the definition of $\Delta_{k+1}$ and Lemma 2, it is easy to check that $\Delta_{k+1}=$ Out $t^{k+1}\left(q_{2}, F_{A g}^{\prime}\right)$.

Claim 3 For any $\sigma_{2} \in \operatorname{Out}\left(q_{2}, F_{A g}^{\prime}\right)$, there exists $\sigma_{1} \in \operatorname{Out}\left(q_{1}, F_{A g}\right)$ such that $\sigma_{1} \sim_{A g}^{\varepsilon} \sigma_{2}$.

Suppose that $\sigma_{2} \in \operatorname{Out}\left(q_{2}, F_{A g}^{\prime}\right)$. By Definition 3 and Claim 2, for any $n \in \mathbb{N}, \sigma_{2}[1, n] \in \operatorname{Out}^{n}\left(q_{2}, F_{A q}^{\prime}\right)=\Delta_{n}$. In order to demonstrate the existence of the desired $\sigma_{1} \in \operatorname{Out}\left(q_{1}, F_{A g}\right)$, we construct the tree

$$
<\bigcup_{n \in \mathbb{N}}\left\{s_{n} \in \operatorname{Out}^{n}\left(q_{1}, F_{A g}\right): s_{n} \sim_{A g}^{\varepsilon} \sigma_{2}[1, n]\right\}, R, q_{1}>
$$


as:

(a) $q_{1}$ is the root;

(b) for any $s, s^{\prime} \in \bigcup_{n \in \mathbb{N}}\left\{s_{n} \in O u t^{n}\left(q_{1}, F_{A g}\right): s_{n} \sim_{A g}^{\varepsilon} \sigma_{2}[1, n]\right\}, s R s^{\prime}$ if and only if $\left|s^{\prime}\right|=|s|+1$ and $s^{\prime}[1,|s|]=s$.

By Claim 1, for any $n \in \mathbb{N}$, it follows from $\sigma_{2}[1, n] \in \Delta_{n}$ that $\left\{s_{n} \in\right.$ $\left.\operatorname{Out}^{n}\left(q_{1}, F_{A g}\right): s_{n} \sim_{A g}^{\varepsilon} \sigma_{2}[1, n]\right\}$ is non-empty. Thus such tree is infinite. On the other hand, since $T_{2}$ is finite branching, it is easy to see that this tree is finite branching. Therefore, by König's lemma, there exists an infinite branch of this tree. Suppose that this branch is $s_{1}\left(=q_{1}\right), s_{2}, s_{3}, \cdots$. We define $\sigma_{1}$ as $\sigma_{1}[i] \triangleq s_{i}[$ end $]$ for each $i \in \mathbb{N}$. By the construction of this tree, we have $\sigma_{1} \in \operatorname{Out}\left(q_{1}, F_{A g}\right)$ and $\sigma_{1} \sim_{A g}^{\varepsilon} \sigma_{2}$.

Due to symmetry of $\sim_{A g}^{\varepsilon}$, the clauses (1-b) and (2-b) in the lemma below are redundant. However, to prove (1-a) and (2-a) by induction, we need to use induction hypothesis on (1-b) and (2-b), thus, they are also listed explicitly in the lemma.

Lemma 7 Let $T_{i}=\left(S_{i}, \mathbb{P}, \Omega, \Pi_{i}, \hbar_{i}\right)$ be two finite branching alternating transition systems $(i=1,2)$. Suppose that $d$ is a metric over $\mathbb{P}, \varepsilon \in \mathbb{R}_{+}^{0}$ and $A g \subseteq \Omega$. For any $q_{1} \in S_{1}, q_{2} \in S_{2}, \sigma_{1} \in\left(S_{1}\right)^{\omega}$ and $\sigma_{2} \in\left(S_{2}\right)^{\omega}$,

(1) if $q_{1} \sim_{A g}^{\varepsilon} q_{2}$, then for any $(\varphi, \gamma) \in H_{A g}^{\varepsilon}$, (1-a) $\left(T_{1}, d\right), q_{1} \models \varphi \Rightarrow\left(T_{2}, d\right), q_{2} \models \gamma$, (1-b) $\left(T_{2}, d\right), q_{2} \models \varphi \Rightarrow\left(T_{1}, d\right), q_{1} \models \gamma$;

(2) if $\sigma_{1} \sim_{A g}^{\varepsilon} \sigma_{2}$, then for any $(\psi, \phi) \in E_{A g}^{\varepsilon}$, (2-a) $\left(T_{1}, d\right), \sigma_{1} \models \psi \Rightarrow\left(T_{2}, d\right), \sigma_{2} \models \phi$, (2-b) $\left(T_{2}, d\right), \sigma_{2} \models \psi \Rightarrow\left(T_{1}, d\right), \sigma_{1} \models \phi$.

Proof We prove (1) and (2) simultaneously by induction on the ranks of $\varphi$ and $\psi$.

By Definition $8, \xi_{s}(\varphi)>0$ and $\xi_{p}(\psi)>0$ for any state formula $\varphi$ and path formula $\psi$. So it is clear that if $\xi_{s}(\varphi)=0$ and $\xi_{p}(\psi)=0$ then (1) and (2) hold.

Suppose that $(\varphi, \gamma) \in H_{A g}^{\varepsilon},(\psi, \phi) \in E_{A g}^{\varepsilon}, \xi_{s}(\varphi)=\xi_{p}(\psi)=n+1$ and the conclusions (1) and (2) hold for any $\left(\varphi_{0}, \gamma_{0}\right) \in H_{A g}^{\varepsilon}$ and $\left(\psi_{0}, \phi_{0}\right) \in E_{A g}^{\varepsilon}$ with $\xi_{s}\left(\varphi_{0}\right)=\xi_{p}\left(\psi_{0}\right) \leq n$.

(1-a) By Lemma 4, $\varphi$ is in one of the following forms: $p, \neg \gamma_{1}, \varphi_{1} \wedge \varphi_{2}$, and $\langle\langle A g\rangle\rangle \psi_{1}$. The argument is split into four cases based on the form of $\varphi$. In the following, we just consider some sample cases.

Case $1.1 \varphi=p$ for some $p \in \mathbb{P}$. Suppose that $q_{1} \sim{ }_{A g}^{\varepsilon} q_{2}$ and $\left(T_{1}, d\right), q_{1} \models \varphi$. By Lemma 4, we have $\gamma=\langle\varepsilon\rangle p$. Then, since $\left(T_{1}, d\right), q_{1} \models \varphi$ and $q_{1} \sim_{A g}^{\varepsilon} q_{2}$, it follows from Definition 7 and Theorem 1 that $\Pi_{1}\left(q_{1}\right)=p$ and $d\left(p, \Pi_{2}\left(q_{2}\right)\right) \leq \varepsilon$. Further, by Definition 7, we get $\left(T_{2}, d\right), q_{2} \models \gamma$.

Case $1.2 \varphi=\neg \gamma_{0}$. Suppose that $q_{1} \sim_{A g}^{\varepsilon} q_{2}$ and $\left(T_{1}, d\right), q_{1} \models \varphi$. By Lemma 4, there exists a state formula $\varphi_{0}$ such that $\left(\varphi_{0}, \gamma_{0}\right) \in H_{A g}^{\varepsilon}, \gamma=\neg \varphi_{0}$ and $\xi_{s}(\varphi)=\xi_{s}(\gamma)=\xi_{s}\left(\gamma_{0}\right)+1$. It follows from $\left(T_{1}, d\right), q_{1} \models \varphi$ and Definition 7 that $\left(T_{1}, d\right), q_{1} \forall \gamma_{0}$. Further, by induction hypothesis on (1-b), we obtain $\left(T_{2}, d\right), q_{2} \not \models \varphi_{0}$. Therefore, $\left(T_{2}, d\right), q_{2} \models \gamma$. 
Case $1.3 \varphi=\langle\langle A g\rangle\rangle \psi$. Suppose that $q_{1} \sim_{A g}^{\varepsilon} q_{2}$ and $\left(T_{1}, d\right), q_{1} \models \varphi$. It follows from Lemma 4 that there exists a path formula $\phi$ such that $(\psi, \phi) \in E_{A g}^{\varepsilon}$, $\gamma=\langle\langle A g\rangle\rangle \phi$ and $\xi_{s}(\varphi)=\xi_{s}(\gamma)=\xi_{p}(\psi)+1$. Due to $\left(T_{1}, d\right), q_{1} \models\langle\langle A g\rangle\rangle \psi$ and Definition 7 there is a strategy $F_{A g}$ of $A g$ such that for any $\sigma \in O u t\left(q_{1}, F_{A g}\right)$, $\left(T_{1}, d\right), \sigma \models \psi$. It follows from $q_{1} \sim_{A g}^{\varepsilon} q_{2}$ and Lemma 6 that there exists a strategy $F_{A g}^{\prime}:\left(S_{2}\right)^{+} \rightarrow 2^{S_{2}}$ such that

for any $\sigma^{\prime} \in \operatorname{Out}\left(q_{2}, F_{A g}^{\prime}\right), \sigma \sim_{A g}^{\varepsilon} \sigma^{\prime}$ for some $\sigma \in O u t\left(q_{1}, F_{A g}\right)$.

Then since $\left(T_{1}, d\right), \sigma \models \psi$ for any $\sigma \in O u t\left(q_{1}, F_{A g}\right)$, by induction hypothesis on $(2-a),\left(T_{2}, d\right), \sigma^{\prime} \models \phi$ for any $\sigma^{\prime} \in \operatorname{Out}\left(q_{2}, F_{A g}^{\prime}\right)$. So we have $\left(T_{2}, d\right), q_{2} \models \gamma$.

(2-a) By Lemma 4 again, $\psi$ is in the form of $\varphi_{1}, \neg \phi_{1}, \psi_{1} \wedge \psi_{2}, \mathbf{X} \psi_{1}$ or $\psi_{1} \mathbf{U} \psi_{2}$. We distinguish five cases based on the form of $\psi$ and just consider some sample cases below.

Case 2.1 $\psi$ is a state formula. Suppose that $\sigma_{1} \sim_{A g}^{\varepsilon} \sigma_{2}$ and $\left(T_{1}, d\right), \sigma_{1} \models \psi$. By Lemma 4. $(\psi, \phi) \in H_{A g}^{\varepsilon}$ and $\xi_{p}(\psi)=\xi_{p}(\phi)=\xi_{s}(\psi)+1$. By Definition 7 we have $\left(T_{1}, d\right), \sigma_{1}[1] \models \psi_{1}$. So by induction hypothesis on $(1-a),\left(T_{2}, d\right), \sigma_{2}[1] \models \phi$. Further, it follows from Definition 7 that $\left(T_{2}, d\right), \sigma_{2} \models \phi$.

Case 2.2 $\psi=\psi_{1} \mathbf{U} \psi_{2}$. Suppose that $\sigma_{1} \sim_{A g}^{\varepsilon} \sigma_{2}$ and $\left(T_{1}, d\right), \sigma_{1} \models \psi$. By Lemma 4, there exist path formulas $\phi_{1}$ and $\phi_{2}$ such that $\left(\psi_{i}, \phi_{i}\right) \in E_{A g}^{\varepsilon}(i=$ $1,2), \phi=\phi_{1} \mathbf{U} \phi_{2}$ and $\xi_{p}(\phi)=\xi_{p}(\psi)=\max \left\{\xi_{p}\left(\psi_{1}\right), \xi_{p}\left(\psi_{2}\right)\right\}+1$. Due to $\left(T_{1}, d\right), \sigma_{1} \models \psi_{1} \mathbf{U} \psi_{2}$ and Definition 7 there exists $i \in \mathbb{N}$ such that

$$
\left(T_{1}, d\right), \sigma_{1}[i, \infty] \models \psi_{2} \text { and for any } j<i,\left(T_{1}, d\right), \sigma_{1}[j, \infty] \models \psi_{1} \text {. }
$$

By induction hypothesis on $(2-a)$, it follows that $\left(T_{2}, d\right), \sigma_{2}[i, \infty] \models \phi_{2}$ and for any $j<i,\left(T_{2}, d\right), \sigma_{2}[j, \infty] \models \phi_{1}$. Then by Definition [7, we obtain $\left(T_{2}, d\right), \sigma_{2} \models$ $\phi$.

Lemma 8 Let $T_{i}=\left(S_{i}, \mathbb{P}, \Omega, \Pi_{i}, \hbar_{i}\right)$ be two finite branching alternating transition systems $(i=1,2)$. Suppose that $d$ is a metric over $\mathbb{P}, \varepsilon \in \mathbb{R}_{+}^{0}$ and $A g \subseteq \Omega$. For any $q_{1} \in S_{1}$ and $q_{2} \in S_{2}, q_{1} \sim_{A g}^{\varepsilon} q_{2}$ if they satisfy the following two conditions:

(1) for any $(\varphi, \gamma) \in H_{A g}^{\varepsilon},\left(T_{1}, d\right), q_{1} \models \varphi \Rightarrow\left(T_{2}, d\right), q_{2} \models \gamma$,

(2) for any $(\varphi, \gamma) \in H_{A g}^{\varepsilon},\left(T_{2}, d\right), q_{2} \models \varphi \Rightarrow\left(T_{1}, d\right), q_{1} \models \gamma$.

Proof Set

$R=\left\{\left(q_{1}, q_{2}\right): q_{1} \in S_{1}\right.$ and $q_{2} \in S_{2}$ satisfy the above conditions (1) and (2) $\}$.

To complete the proof, it is enough to show that $R$ is an $(A g, \varepsilon)$-alternating approximate bisimulation. Suppose that $R$ is not. Since each pair in $R$ satisfies the condition (1) in Definition 5, there exists $\left(q_{1}, q_{2}\right) \in R$ satisfying one of the following conditions:

(i) $\exists Q_{1} \in \hbar_{1}\left(q_{1}, A g\right) \forall Q_{2} \in \hbar_{2}\left(q_{2}, A g\right) \exists Q_{2}^{\prime} \in \hbar_{2}\left(q_{2}, \Omega-A g\right) \forall Q_{1}^{\prime} \in \hbar_{1}\left(q_{1}, \Omega-\right.$ $A g)\left(\left(Q_{1} \cap Q_{1}^{\prime}\right) \times\left(Q_{2} \cap Q_{2}^{\prime}\right) \not R R\right)$

(ii) $\exists Q_{2} \in \hbar_{2}\left(q_{2}, A g\right) \forall Q_{1} \in \hbar_{1}\left(q_{1}, A g\right) \exists Q_{1}^{\prime} \in \hbar_{1}\left(q_{1}, \Omega-A g\right) \forall Q_{2}^{\prime} \in \hbar_{2}\left(q_{2}, \Omega-\right.$ $A g)\left(\left(Q_{1} \cap Q_{1}^{\prime}\right) \times\left(Q_{2} \cap Q_{2}^{\prime}\right) \nsubseteq \mathbb{R}\right)$.

W.l.o.g, suppose that (i) holds. It follows from (i) and Definition 1 that for any $Q_{2} \in \hbar_{2}\left(q_{2}, A g\right)$, there exists $Q_{2}^{\prime} \in \hbar_{2}\left(q_{2}, \Omega-A g\right)$ and $q_{2}^{\prime} \in Q_{2}$ such that $\left\{q_{2}^{\prime}\right\}=Q_{2} \cap Q_{2}^{\prime}$ and for any $Q_{1}^{\prime} \in \hbar\left(q_{1}, \Omega-A g\right)$, 


$$
\left\{q_{1}^{\prime}\right\}=Q_{1} \cap Q_{1}^{\prime} \text { and }\left(q_{1}^{\prime}, q_{2}^{\prime}\right) \notin R \text { for unique } q_{1}^{\prime} \text {. }
$$

Hence, for each $Q_{2} \in \hbar_{2}\left(q_{2}, A g\right)$ and $Q_{1}^{\prime} \in \hbar_{1}\left(q_{1}, \Omega-A g\right)$, we can fix a pair of states $q_{Q_{2}} \in Q_{2}$ and $q_{Q_{1}^{\prime}} \in Q_{1}$ satisfying the above conditions. Then for any $Q_{2} \in \hbar_{2}\left(q_{2}, A g\right)$ and $Q_{1}^{\prime} \in \hbar_{1}\left(q_{1}, \Omega-A g\right)$, it follows from $\left(q_{Q_{1}^{\prime}}, q_{Q_{2}}\right) \notin R$ that there exists $(\varphi, \gamma) \in H_{A g}^{\varepsilon}$ such that

$$
\begin{aligned}
& \text { (a) }\left(T_{1}, d\right), q_{Q_{1}^{\prime}} \models \varphi \text { but }\left(T_{2}, d\right), q_{Q_{2}} \models \neg \gamma \text {, or } \\
& \text { (b) }\left(T_{2}, d\right), q_{Q_{2}} \models \varphi \text { but }\left(T_{1}, d\right), q_{Q_{1}^{\prime}} \models \neg \gamma \text {. }
\end{aligned}
$$

If (a) holds, then $\left(T_{2}, d\right), q_{Q_{2}} \models \neg \gamma$ but $\left(T_{1}, d\right), q_{Q_{1}^{\prime}} \models \neg \neg \varphi$. Moreover, by Definition 9, we get $(\neg \gamma, \neg \varphi) \in H_{A g}^{\varepsilon}$. Thus for any $Q_{2} \in \hbar_{2}\left(q_{2}, A g\right)$ and $Q_{1}^{\prime} \in \hbar_{1}\left(q_{1}, \Omega-A g\right)$, there exists $\left(\varphi_{Q_{1}^{\prime}, Q_{2}}, \gamma_{Q_{1}^{\prime}, Q_{2}}\right) \in H_{A g}^{\varepsilon}$ such that

$$
\left(T_{2}, d\right), q_{Q_{2}} \models \varphi_{Q_{1}^{\prime}, Q_{2}} \text { but }\left(T_{1}, d\right), q_{Q_{1}^{\prime}} \models \neg \gamma_{Q_{1}^{\prime}, Q_{2}} .
$$

So, for any $Q_{2}^{*} \in \hbar_{2}\left(q_{2}, A g\right)$, it follows that

$$
\left(T_{2}, d\right), q_{Q_{2}^{*}} \models \bigvee_{Q_{2} \in \hbar_{2}\left(q_{2}, A g\right)} \wedge_{Q_{1}^{\prime} \in \hbar_{1}\left(q_{1}, \Omega-A g\right)} \varphi_{Q_{1}^{\prime}, Q_{2}} \text {. }
$$

By Lemma 1 for any strategy $F_{A g}^{\prime}:\left(S_{2}\right)^{+} \rightarrow 2^{S_{2}}$, we have $F_{A g}^{\prime}\left(q_{2}\right) \in \hbar_{2}\left(q_{2}, A g\right)$ and then it follows from Definition 3 that for some $\sigma_{2} \in \operatorname{Out}\left(q_{2}, F_{A g}^{\prime}\right)$,

$$
\sigma_{2}[2]=q_{F_{A g}^{\prime}\left(q_{2}\right)} \text { and }\left(T_{2}, d\right), \sigma_{2}[2] \models \bigvee_{Q_{2} \in \hbar_{2}\left(q_{2}, A g\right)} \bigwedge_{Q_{1}^{\prime} \in \hbar_{1}\left(q_{1}, \Omega-A g\right)} \varphi_{Q_{1}^{\prime}, Q_{2}} .
$$

Further, by Definition 7 we obtain

$$
\left(T_{2}, d\right), q_{2} \models \neg\langle\langle A g\rangle\rangle \mathbf{X} \bigvee_{Q_{2} \in \hbar_{2}\left(q_{2}, A g\right)} \bigwedge_{Q_{1}^{\prime} \in \hbar_{1}\left(q_{1}, \Omega-A g\right)} \varphi_{Q_{1}^{\prime}, Q_{2}}
$$

Moreover, it follows from Definition 9 that $\left(\varphi^{*}, \gamma^{*}\right) \in H_{A g}^{\varepsilon}$, where

$$
\varphi^{*}=\neg\langle\langle A g\rangle\rangle \neg \mathbf{X} \bigvee_{Q_{2} \in \hbar_{2}\left(q_{2}, A g\right)} \bigwedge_{Q_{1}^{\prime} \in \hbar_{1}\left(q_{1}, \Omega-A g\right)} \varphi_{Q_{1}^{\prime}, Q_{2}}
$$

and

$$
\gamma^{*}=\neg\langle\langle A g\rangle\rangle \neg \mathbf{X} \bigvee_{Q_{2} \in \hbar_{2}\left(q_{2}, A g\right)} \bigwedge_{Q_{1}^{\prime} \in \hbar_{1}\left(q_{1}, \Omega-A g\right)} \gamma_{Q_{1}^{\prime}, Q_{2}}
$$

Hence, due to $\left(T_{2}, d\right), q_{2} \models \varphi^{*}$ and $\left(q_{1}, q_{2}\right) \in R$, we get

$$
\left(T_{1}, d\right), q_{1} \models \gamma^{*} .
$$

On the other hand, by Definition 2, it is clear that $F_{A g}\left(q_{1}\right)=Q_{1} \in$ $\hbar_{1}\left(q_{1}, A g\right)$ for some strategy $F_{A g}:\left(S_{1}\right)^{+} \rightarrow 2^{S_{1}}$. Then by (2), there exists $\sigma_{1} \in \operatorname{Out}\left(q_{1}, F_{A g}\right)$ such that

$$
\left(T_{1}, d\right), \sigma_{1}[2] \models \bigvee_{Q_{2} \in \hbar_{2}\left(q_{2}, A g\right)} \bigwedge_{Q_{1}^{\prime} \in \hbar_{1}\left(q_{1}, \Omega-A g\right)} \gamma_{Q_{1}^{\prime}, Q_{2}} .
$$

By Definition 3. $\left\{\sigma_{1}[2]\right\}=Q_{1} \cap Q_{1}^{\prime \prime}$ for some $Q_{1}^{\prime \prime} \in \hbar_{1}\left(q_{1}, \Omega-A g\right)$. Clearly, $\sigma_{1}[2]=q_{Q_{1}^{\prime \prime}}$. Then $\left(T_{1}, d\right), q_{Q_{1}^{\prime \prime}} \models \bigwedge_{Q_{1}^{\prime} \in \hbar_{1}\left(q_{1}, \Omega-A g\right)} \gamma_{Q_{1}^{\prime}, Q_{2}^{*}}$ for some $Q_{2}^{*} \in$ $\hbar_{2}\left(q_{2}, A g\right)$. Thus we have $\left(T_{1}, d\right), q_{Q_{1}^{\prime \prime}} \models \gamma_{Q_{1}^{\prime \prime}, Q_{2}^{*}}$, which contradicts (1).

\footnotetext{
${ }^{3} \bigvee_{i \in I} \varphi_{i}$ and $\bigwedge_{i \in I} \varphi_{i}$ can be defined as usual, where $I$ is a finite index set.
} 
Now, we arrive at the main result of this section, which offers a logical characterization of alternating approximate bisimilariy.

Theorem 2 Let $T_{i}=\left(S_{i}, \mathbb{P}, \Omega, \Pi_{i}, \hbar_{i}\right)$ be two finite branching alternating transition systems $(i=1,2)$. Suppose that $d$ is a metric over $\mathbb{P}, \varepsilon \in \mathbb{R}_{+}^{0}$ and $\mathrm{Ag} \subseteq \Omega$. For any $q_{1} \in S_{1}$ and $q_{2} \in S_{2}, q_{1} \sim_{A g}^{\varepsilon} q_{2}$ if and only if they satisfy the following conditions:

(1) for any $(\varphi, \gamma) \in H_{A g}^{\varepsilon},\left(T_{1}, d\right), q_{1} \models \varphi \Rightarrow\left(T_{2}, d\right), q_{2} \models \gamma$,

(2) for any $(\varphi, \gamma) \in H_{A g}^{\varepsilon},\left(T_{2}, d\right), q_{2} \models \varphi \Rightarrow\left(T_{1}, d\right), q_{1} \models \gamma$.

Proof Immediately follows from Lemma 7 and 8

For $\varepsilon=0$, by the clause (1) in Lemma 3 , $(A g, \varepsilon)$-alternating approximate bisimilarity is an equivalence relation. In this case, the above result degenerates into one in the usual style.

Corollary 1 Let $T_{i}=\left(S_{i}, \mathbb{P}, \Omega, \Pi_{i}, \hbar_{i}\right)$ be two finite branching alternating transition systems $(i=1,2)$. Suppose that $d$ is a metric over $\mathbb{P}$ and $A g \subseteq \Omega$. For any $q_{1} \in S_{1}$ and $q_{2} \in S_{2}, q_{1} \sim_{A g}^{0} q_{2}$ if and only if for any Ag-ATL $L_{0}$ state formula $\varphi$,

$$
\left(T_{1}, d\right), q_{1} \models \varphi \Leftrightarrow\left(T_{2}, d\right), q_{2} \models \varphi .
$$

Proof Suppose that $q_{1} \in S_{1}$ and $q_{2} \in S_{2}$. Then we have

$$
q_{1} \sim{ }_{A g}^{0} q_{2}
$$

iff for any $(\varphi, \gamma) \in H_{A g}^{0},\left(T_{1}, d\right), q_{1} \models \varphi \Rightarrow\left(T_{2}, d\right), q_{2} \models \gamma$, and vice verse (by Theorem 2)

iff for any $(\varphi, \gamma) \in H_{A g}^{0},\left(T_{1}, d\right), q_{1} \models \varphi \Rightarrow\left(T_{2}, d\right), q_{2} \models \varphi$, and vice verse (by (1) in Lemma 5

iff for any $(\varphi, \gamma) \in H_{A g}^{0},\left(T_{1}, d\right), q_{1} \models \varphi \Leftrightarrow\left(T_{2}, d\right), q_{2} \models \varphi$

iff for any $A g$-ATL 0 state formula $\varphi,\left(T_{1}, d\right), q_{1} \models \varphi \Leftrightarrow\left(T_{2}, d\right), q_{2} \models \varphi$.

(by (3) in Lemma 5)

As mentioned in Section 2, a logical characterization of alternating bisimilarity in terms of ATL has been provided in 3 . In the following, we show that this characterization can be obtained from the above result immediately. The syntax and semantic of ATL is similar to $\mathrm{ATL}_{\varepsilon}$ and the only difference between them is that ATL does not refer to the modal operator $\langle\varepsilon\rangle$ and metric over observations. Here we do not recall ATL formally, which can be found in [2] [3]. Since the semantics of ATL has nothing to do with metric over observations, we use $T, q \models \varphi$ to denote that the state $q$ of $T$ satisfies ATL formula $\varphi$.

Corollary 2 Let $T_{i}=\left(S_{i}, \mathbb{P}, \Omega, \Pi_{i}, \hbar_{i}\right)$ be two finite branching alternating transition systems $(i=1,2)$ and $A g \subseteq \Omega$. For any $q_{1} \in S_{1}$ and $q_{2} \in S_{2}$, $q_{1} \sim_{A g} q_{2}$ if and only if for any Ag-ATL state formula $\varphi$,

$$
T_{1}, q_{1} \models \varphi \Leftrightarrow T_{2}, q_{2} \models \varphi .
$$

Proof It is not difficult to see that two states are logical equivalent w.r.t $A g$ $\mathrm{ATL}_{0}$ state formulas if and only if so are they w.r.t $\mathrm{Ag}$-ATL state formulas. Thus by the clause (1) in Lemma 3 and Corollary 1 the conclusion holds. 


\section{Application of modal characterization: temporal logical control}

For control systems with disturbances, Pola and Tabuada adopt infinite alternating transition systems to model their sampling systems and construct finite alternating transition systems as their finite abstractions 25] 26. In these work, alternating approximate bisimilarity is introduced to capture the equivalence between these sampling systems and finite abstractions. Based on results obtained in the previous section, we will establish a relationship between linear temporal logical specifications which are satisfied by these sampling systems under control and by the corresponding finite abstractions under control, respectively. Moreover, we give a potential application of this result in the linear temporal logical control of control systems with disturbances.

\subsection{Control systems and its finite abstractions}

This subsection recalls some notions and results about control systems with disturbances and their finite abstractions provided by [25] [26]. Before doing so, we introduce some useful notations.

Given a vector $x \in \mathbb{R}^{n}$, we denote by $x_{i}$ the $i$-th element of $x$ and $\|x\| \triangleq$ $\max \left\{\left|x_{1}\right|,\left|x_{2}\right|, \cdots,\left|x_{n}\right|\right\}$ where $\left|x_{i}\right|$ is the absolute value of $x_{i}$. The set $X \subseteq$ $\mathbb{R}^{n}$ is said to be bounded if and only if $\sup \{\|x\|: x \in X\}<\infty$. For any measurable function $f: \mathbb{R}_{+}^{0} \rightarrow \mathbb{R},\|f\|_{\infty} \triangleq \sup \{\|f(t)\|, t \geq 0\}$ and $f$ is said to be essentially bounded if $\|f\|_{\infty}<\infty$. For a given time $\tau \in \mathbb{R}_{+}$, define $f_{\tau}$ so that $f_{\tau}(t)=f(t)$ for any $t \in[0, \tau)$, and $f(t)=0$ elsewhere; $f$ is said to be locally essentially bounded if for any $\tau \in \mathbb{R}_{+}, f_{\tau}$ is essentially bounded. In this section, we consider the metric $d$ on $\mathbb{R}^{n}$ defined as $d(x, y)=\max \left\{\mid x_{1}-\right.$ $\left.y_{1}|,| x_{2}-y_{2}|, \cdots| x_{n}-y_{n} \mid\right\}$.

Definition 10 25 26 A control system with disturbances is a quadruple $\Sigma=(X, W, \mathcal{W}, f)$, where

- $X \subseteq \mathbb{R}^{n}$ is the state space;

- $W=U \times V$ is the input space, where

$U \subseteq \mathbb{R}^{m}$ is the control input space;

$V \subseteq \mathbb{R}^{s}$ is the disturbance input space;

- $\mathcal{W}$ is a subset of the set of all measurable and locally essentially bounded functions of time from intervals of the form $] a, b[\subseteq \mathbb{R}$ to $W$ with $a<0$ and $b>0$

- $f: X \times W \rightarrow X$ is a continuous map satisfying the following Lipschitz assumption: for every compact set $K \subset X$, there exists a constant $\kappa>0$ such that

$$
\|f(x, w)-f(y, w)\| \leq \kappa\|x-y\|
$$

for all $x, y \in K$ and all $w \in W$.

A locally absolutely continuous curve $\mathbf{x}:] a, b[\rightarrow X$ is said to be a trajectory if there exists $\mathbf{w} \in \mathcal{W}$ satisfying $\dot{\mathbf{x}}(t)=f(\mathbf{x}(t), \mathbf{w}(t))$ for almost all $t \in] a, b[$.

A control system is said to be forward complete if and only if every trajectory is defined on an interval of the form $] a, \infty[$. 
Convention. As in [25] [26], we assume that $0 \in X, X \subseteq \mathbb{R}^{n}$ is a bounded polytopic sets with non-empty interior, and the control system $\Sigma$ is forward complete.

For such systems, Pola and Tabuada adopt a variety of alternating transition systems as models of their sampling systems and finite abstractions [25] 26].

Definition 11 An alternating transition system is a tuple $T=(S, A, B, \longrightarrow$ $, \mathbb{P}, \Pi)$ consisting of a set of states $S$, a set of control labels $A$, a set of disturbance labels $B$, a transition relation $\rightarrow \subseteq S \times A \times B \times S$, an observation set $\mathbb{P}$ and an observation function $\Pi: S \rightarrow \mathbb{P}$.

We say that an alternating transition system $T$ is metric if the observation set $\mathbb{P}$ is equipped with a metric, $T$ is non-blocking if $\left\{q^{\prime}: q \stackrel{a, b}{\longrightarrow} q^{\prime}\right\} \neq \emptyset$ for any $q \in Q, a \in A$ and $b \in B$, and $T$ is finite if $S, A$ and $B$ are finite. An infinite sequence $\sigma \in S^{\omega}$ is said to be a trajectory of $T$ if and only if for all $i \in \mathbb{N}$, $\sigma[i] \stackrel{a_{i}, b_{i}}{\longrightarrow} \sigma[i+1]$ for some $a_{i} \in A$ and $b_{i} \in B$.

We may view the above alternating transition system as a variant of one defined in Definition 1. The differences between them lie in: the above notion involves only two agents which choose successor states by means of choosing inputs, moreover, successor states of a given state may not be determined even if these two agents make choices. In this section, following Pola and Tabuada, the notion "alternating transition system" refers to the one defined above. Similar to Definition 2 and 3 , the strategy and the corresponding outcomes of these systems are defined below.

Definition 12 A control strategy for an alternating transition system $T=$ $(S, A, B, \longrightarrow, \mathbb{P}, \Pi)$ is a function $F: S^{+} \rightarrow 2^{A}-\{\emptyset\}$. For any $q \in S$, the outcomes $\operatorname{Out}_{T}^{n}(q, F)(n \in \mathbb{N})$ and $\operatorname{Out}_{T}(q, F)$ of $F$ from $q$ are defined as follows:

$$
\begin{aligned}
& O u t_{T}^{n}(q, F)=\left\{s \in S^{n}: s[1]=q\right. \text { and } \\
& \left.\forall 1 \leq i<n \exists a_{i} \in F(s[1, i]) \exists b_{i} \in B\left(s[i] \stackrel{a_{i}, b_{i}}{\longrightarrow} s[i+1]\right)\right\}, \\
& \text { Out }_{T}(q, F)=\left\{\sigma \in S^{\omega}: \sigma[1]=q\right. \text { and } \\
& \left.\forall i \in \mathbb{N} \exists a_{i} \in F(\sigma[1, i]) \exists b_{i} \in B\left(\sigma[i] \stackrel{a_{i}, b_{i}}{\longrightarrow} \sigma[i+1]\right)\right\} .
\end{aligned}
$$

The notion of alternating approximate bisimilarity provided by Pola and Tabuada is recalled below. It is not difficult to see that such notion and one in Definition [5] are the same in spirit.

Definition 13 [26] Let $T_{i}=\left(S_{i}, A_{i}, B_{i}, \rightarrow_{i}, \mathbb{P}, \Pi_{i}\right)(i=1,2)$ be two metric, non-blocking alternating transition systems and let $d$ be a metric over $\mathbb{P}$. Given a precision $\varepsilon \in \mathbb{R}_{+}$, a relation $R \subseteq S_{1} \times S_{2}$ is said to be an alternating $\varepsilon$-approximate $(A \varepsilon A)$ bisimulation relation between $T_{1}$ and $T_{2}$ if for any $\left(q_{1}, q_{2}\right) \in R$

(i) $d\left(\Pi_{1}\left(q_{1}\right), \Pi_{2}\left(q_{2}\right)\right) \leq \varepsilon$; 
(ii) $\forall a_{1} \in A_{1} \exists a_{2} \in A_{2} \forall b_{2} \in B_{2} \forall q_{2}^{\prime} \in S_{2}\left(q_{2} \stackrel{a_{2}, b_{2}}{\longrightarrow}{ }_{2} q_{2}^{\prime} \Rightarrow \exists b_{1} \in B_{1} \exists q_{1}^{\prime} \in S_{1}\right.$ $\left(q_{1} \stackrel{a_{1}, b_{1}}{\longrightarrow}{ }_{1} q_{1}^{\prime}\right.$ and $\left.\left.\left(q_{1}^{\prime}, q_{2}^{\prime}\right) \in R\right)\right)$.

(iii) $\forall a_{2} \in A_{2} \exists a_{1} \in A_{1} \forall b_{1} \in B_{1} \forall q_{1}^{\prime} \in S_{1}\left(q_{1} \stackrel{a_{1}, b_{1}}{\longrightarrow}{ }_{1} q_{1}^{\prime} \Rightarrow \exists b_{2} \in B_{2} \exists q_{2}^{\prime} \in S_{2}\right.$ $\left(q_{2} \stackrel{a_{2}, b_{2}}{\longrightarrow} 2 q_{2}^{\prime}\right.$ and $\left.\left.\left(q_{1}^{\prime}, q_{2}^{\prime}\right) \in R\right)\right)$.

For any $q_{1} \in S_{1}$ and $q_{2} \in S_{2}$, they are said to be $A \varepsilon A$ bisimilar, in symbols $q_{1} \sim_{\varepsilon} q_{2}$, if there exists an $A \varepsilon A$ bisimulation relation $R$ between $T_{1}$ and $T_{2}$ such that $\left(q_{1}, q_{2}\right) \in R$. Moreover, $T_{1}$ and $T_{2}$ are said to be $A \varepsilon A$ bisimilar, in symbols $T_{1} \simeq_{\varepsilon} T_{2}$, if there exists an $A \varepsilon A$ bisimulation relation $R$ between $T_{1}$ and $T_{2}$ such that $S_{1}=\left\{q_{1} \in S_{1}:\left(q_{1}, q_{2}\right) \in R\right.$ for some $\left.q_{2} \in S_{2}\right\}$ and $S_{2}=\left\{q_{2} \in S_{2}:\left(q_{1}, q_{2}\right) \in R\right.$ for some $\left.q_{1} \in S_{1}\right\}$.

For control systems with disturbances, Pola and Tabuada construct infinite and finite non-blocking alternating transition systems as their samples and finite abstractions, respectively. The detailed construction is referred to [26]. Moreover, they demonstrate that under some assumption, the sample $T_{\tau}(\Sigma)$ and finite abstraction are alternating approximate bisimilar.

Theorem 3 [26] Given a control system $\Sigma=(X, U \times V, \mathcal{W}, f)$, if $\Sigma$ is $\delta$ $G A S$ and $U \times V$ is compact, then for any desired precision $\varepsilon \in \mathbb{R}_{+}$, there exist $\tau \in \mathbb{R}_{+}$and a finite abstraction $T$ of $\Sigma$ that is A $A$ bisimilar to the sampling system $T_{\tau}(\Sigma)$ of $2 \sqrt{4}$.

For convenience, we set $T_{\varepsilon, \tau}(\Sigma) \triangleq\{T: T$ is a finite abstraction of $\Sigma$ that is $A \varepsilon A$ bisimilar to the sampling system $\left.T_{\tau}(\Sigma)\right\}$.

\subsection{Logical specifications satisfied by samples and abstractions}

In recent years, temporal logic, due to its resemblance to natural language and the existence of algorithms for model checking, is widely adopted to describe the desired specifications of control systems. For example, linear temporal logic (LTL) is used to express specifications of discrete-time linear systems [8] and continuous-time linear systems [7]. On the other hand, as mentioned in Introduction, finite abstractions of control systems often are adopted to the analysis and design of control systems. Then a natural question arises at this point: what is the relationship between linear temporal logical specifications which are satisfied under control by sampling systems and by the corresponding finite abstractions respectively? This subsection intends to consider such question. To this end, we introduce linear temporal logic $\operatorname{LTL}_{+}^{\varepsilon}$ as follows.

Definition 14 Let $\mathbb{P}$ be a finite set of propositions and $\varepsilon \in \mathbb{R}_{+}$. LTL $L_{+}^{\varepsilon}(\mathbb{P})$ formulas are defined inductively as:

$$
\phi::=p|\langle\varepsilon\rangle p| \phi_{1} \vee \phi_{2}\left|\phi_{1} \wedge \phi_{2}\right| \mathbf{X} \phi \mid \phi_{1} \mathbf{U} \phi_{2} \text {, where } p \in \mathbb{P} \text {. }
$$

For any $\operatorname{LTL}_{+}^{\varepsilon}(\mathbb{P})$ formula $\phi$, if $\langle\varepsilon\rangle$ does not occur in $\phi$, then $\phi$ is said to be a $\mathrm{LTL}_{+}(\mathbb{P})$ formula.

\footnotetext{
4 The definition of $\delta$-GAS can be found in 26 .
} 
As usual, if $\mathbb{P}$ is clear from the context, we abbreviate $\mathrm{LTL}_{+}^{\varepsilon}(\mathbb{P})\left(\mathrm{LTL}_{+}(\mathbb{P})\right)$ to $\mathrm{LTL}_{+}^{\varepsilon}\left(\mathrm{LTL}_{+}\right.$, respectively).

Definition 15 Let $T=(S, A, B, \rightarrow, \mathbb{P}, \Pi)$ be an alternating transition system, $\varepsilon \in \mathbb{R}_{+}$and let $d$ be a metric over $\mathbb{P}$. The satisfaction relation $\models \subseteq$ $S^{\omega} \times L T L_{+}^{\varepsilon}(\mathbb{P})$ is inductively defined as:

- $(T, d), \sigma \models p$ iff $\Pi(\sigma[1])=p$;

- $(T, d), \sigma \models\langle\varepsilon\rangle p$ iff $d(p, \Pi(\sigma[1])) \leq \varepsilon$;

- $(T, d), \sigma \models \phi_{1} \vee \phi_{2}$ iff $(T, d), \sigma \models \phi_{1}$ or $(T, d), \sigma \models \phi_{2}$;

- $(T, d), \sigma \models \phi_{1} \wedge \phi_{2}$ iff $(T, d), \sigma \models \phi_{1}$ and $(T, d), \sigma \models \phi_{2}$;

- $(T, d), \sigma \models \mathbf{X} \phi$ iff $(T, d), \sigma[2, \infty] \models \phi$;

- $(T, d), \sigma \models \phi_{1} \mathbf{U} \phi_{2}$ iff there exists $j \in \mathbb{N}$ such that $(T, d), \sigma[j, \infty] \models \phi_{2}$ and for any $i<j,(T, d), \sigma[i, \infty] \models \phi_{1}$.

Obviously, $\mathrm{LTL}_{+}^{\varepsilon}$ can be viewed as a sublanguage of $\mathrm{ATL}_{\varepsilon}$. In particular, by the above definitions and Definition 6 and 7 , each $\mathrm{LTL}_{+}^{\varepsilon}$ formula can be seen as a path formula of $\mathrm{ATL}_{\varepsilon}$.

Inspired by Definition 9, we introduce a transformation function below.

Definition 16 Let $\mathbb{P}$ be a set of propositions and $\varepsilon \in \mathbb{R}_{+}$. The transformation $\operatorname{Tr}_{\varepsilon}$ mapping $\mathrm{LTL}_{+}$formulas to $\mathrm{LTL}_{+}^{\varepsilon}$ formulas is inductively defined as follows:

- $\operatorname{Tr}_{\varepsilon}(p)=\langle\varepsilon\rangle p$ for any $p \in \mathbb{P}$;

- $T r_{\varepsilon}\left(\phi_{1} \vee \phi_{2}\right)=T r_{\varepsilon}\left(\phi_{1}\right) \vee T r_{\varepsilon}\left(\phi_{2}\right)$;

- $\operatorname{Tr}_{\varepsilon}\left(\phi_{1} \wedge \phi_{2}\right)=\operatorname{Tr} \varepsilon\left(\phi_{1}\right) \wedge \operatorname{Tr} r_{\varepsilon}\left(\phi_{2}\right) ;$

- $\operatorname{Tr}_{\varepsilon}(\mathbf{X} \phi)=\mathbf{X} \operatorname{Tr}_{\varepsilon}(\phi)$;

- $\operatorname{Tr}_{\varepsilon}\left(\phi_{1} \mathbf{U} \phi_{2}\right)=\operatorname{Tr}_{\varepsilon}\left(\phi_{1}\right) \mathbf{U} T r_{\varepsilon}\left(\phi_{2}\right)$.

Clearly, the graph of such transformation is a subrelation of $E_{A g}^{\varepsilon}$ (see Definition (9), that is

Proposition 3 For any $L T L_{+}$formula $\phi,\left(\phi, \operatorname{Tr}_{\varepsilon}(\phi)\right) \in E_{A g}^{\varepsilon}$.

Proof Follows from Definition 9 and 16

Then, by Proposition 2, for each pair of formulas $\phi$ and $\operatorname{Tr}_{\varepsilon}(\phi)$, if a state sequence satisfies $\phi$ then it satisfies $\operatorname{Tr}_{\varepsilon}(\phi)$. But the converse of this result fails in general. So, given $\phi$ and $\operatorname{Tr}_{\varepsilon}(\phi)$, when considering them as specifications, we may view $\operatorname{Tr}_{\varepsilon}(\phi)$ as a looser version of specification $\phi$.

Similar to Lemmal7 we may prove the following result. It should be pointed out that although there exist some differences between notions involved in Lemma 7 and Proposition 4 (see Definition 1 and 11, Definition 5 and 13), the latter may be proved analogously to the former.

Proposition 4 Let $T_{1}=\left(S_{1}, A_{1}, B_{1}, \rightarrow_{1}, \mathbb{P}, \Pi_{1}\right)$ be an infinite, non-blocking alternating transition system and $T_{2}=\left(S_{2}, A_{2}, B_{2}, \rightarrow_{2}, \mathbb{P}, \Pi_{2}\right)$ be a finite, nonblocking alternating transition system. Suppose that $d$ is a metric over $\mathbb{P}$ and $\varepsilon \in \mathbb{R}_{+}$. For any $q_{1} \in S_{1}$ and $q_{2} \in S_{2}$ with $q_{1} \sim_{\varepsilon} q_{2}$ and for any $L T L_{+}$formula $\phi$, if there exists a strategy $F: S^{+} \rightarrow 2^{A_{2}}$ of $T_{2}$ such that $\left(T_{2}, d\right), \sigma \models \phi$ for any $\sigma \in \operatorname{Out}\left(q_{2}, F\right)$, then so does $T_{1}$ for $\operatorname{Tr}_{\varepsilon}(\phi)$. 


\begin{tabular}{|c|c|c|c|}
\hline & Control system & $\begin{array}{l}\text { Model of control } \\
\text { system }\end{array}$ & Finite abstraction \\
\hline Plant & Linear system $\Sigma$ & $\begin{array}{l}\text { Infinite transition } \\
\text { system } T_{\Sigma}\end{array}$ & $\stackrel{\text { bisimilar }}{\stackrel{\text { Finite transition }}{\text { system } T_{\Sigma}^{f}}}$ \\
\hline Specification & LTL formula $\varphi_{0}$ & LTL formula $\varphi_{0}$ & LTL formula $\varphi_{0}$ \\
\hline Controller & $\begin{array}{l}\text { Close-loop system } H \\
\text { satisfying } \varphi_{0}\end{array}$ & $\begin{array}{l}\text { Controller } T_{c} \text { of } T_{\Sigma} \\
\text { enforcing } \varphi_{0}\end{array}$ & Controller $T_{\mathrm{c}}$ of $T_{\Sigma}^{f}$ enforcing $\varphi_{0}$ \\
\hline
\end{tabular}

Fig. 1 Controller design [30]: non-disturbance case

Immediately, we have

Corollary 3 Let $\Sigma=(X, U \times V, \mathcal{W}, f)$ be a $\delta$-GAS control system with compact input space $U \times V$ and $\varepsilon \in \mathbb{R}_{+}$. Then there exists $\tau \in \mathbb{R}_{+}$and a finite

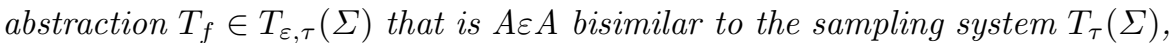
and for any $T \in T_{\varepsilon, \tau}(\Sigma)$ and $L T L_{+}$formula $\phi$, if there exists a state $q$ and $a$ strategy $F$ of $T$ such that $(T, d), \sigma \models \phi$ for any $\sigma \in O$ Out $(q, F)$, then so does $T_{\tau}(\Sigma)$ for $\operatorname{Tr}_{\varepsilon}(\phi)$.

Proof Follows from Proposition 4 and Theorem 3.

Due to the above result, for a control system with disturbances satisfying the conditions mentioned in Corollary 3 , given a LTL formula $\phi$ as a specification, if its finite abstraction and its sampling system are $A \varepsilon A$ bisimilar and the former satisfies specification $\phi$ under control, then so does the latter for a looser specification $\operatorname{Tr}_{\varepsilon}(\phi)$.

\subsection{Linear temporal logical control}

Recently, finite abstraction and the notion of bisimilarity have attracted some people's notice in the area of analysis and design of control systems [4] 12] 29] 30]. In general, control systems and their finite abstractions share properties of interest if they are bisimilar. In particular, according to modal characterization of bisimilarity, they satisfy same temporal logical properties. Moreover, the analysis and design of finite abstractions is simpler than that of control systems. Thus the analysis and design of control systems can be equivalently performed on the corresponding finite abstractions.

As an example, Fig 1 illustrates the function of finite abstraction and bisimilarity in the formal design of linear discrete system [30. Given a linear discrete system $\Sigma$, Tabuada and Pappas provide an infinite transition system $T_{\Sigma}$ as the formal model of $\Sigma$ and construct a finite transition system $T_{\Sigma}^{f}$ as the finite abstraction of $\Sigma$. They prove the following result which lays the foundation of the design method of controllers presented in [30]. 


\begin{tabular}{|c|c|c|c|}
\hline & Control system & Sampling system & Finite abstraction \\
\hline Plant & Control system $\Sigma<$ & Infinite $\operatorname{ATS~}_{\tau}(\Sigma) \stackrel{[26]}{\square}$ & Finite ATS T \\
\hline Specification & & $\mathrm{LTL}^{\varepsilon}{ }_{+}$formula $\operatorname{Tr}_{\varepsilon}(\phi) \stackrel{\operatorname{Tr}_{\varepsilon}}{I}$ & $\mathrm{LTL}_{+}$formula $\phi$ \\
\hline Controller & $\begin{array}{c}\text { Controller so that } \\
\mathrm{T}_{\tau}(\Sigma) \text { satisfies } \operatorname{Tr}_{\varepsilon}(\phi)\end{array}$ & $\begin{array}{c}\text { Control strategy of }{ }^{\text {Cor } 3} \\
\mathrm{~T}_{\tau}(\Sigma) \text { enforcing } \operatorname{Tr}_{\varepsilon}(\phi)\end{array}$ & $\begin{array}{l}\text { Control strategy of } \mathrm{T} \\
\quad \text { enforcing } \phi\end{array}$ \\
\hline
\end{tabular}

Fig. 2 Controller design : disturbance case

$T_{\Sigma}$ and $T_{\Sigma}^{f}$ are bisimilar and then share the same properties described by linear temporal logic. $(*)$

Thus, given an LTL specification $\varphi_{0}$, the formal design of $T_{\Sigma}$ can be equivalently performed on the finite abstraction $T_{\Sigma}^{f}$. Tabuada and Pappas construct a controller $T_{c}$ of $T_{\Sigma}^{f}$ enforcing $\varphi_{0}$ and demonstrate that $T_{\Sigma}$ satisfies $\varphi_{0}$ under this controller as well. Furthermore, based on this controller, a close-loop system $H$ satisfying $\varphi_{0}$ is generated. Similar methods are also adopted in [12] 21] 29].

It is worth to be pointed out that the work 4, 12, 29] 30. consider only the non-disturbance control systems. We intend to generalize these methods to the disturbance case. Similar to the conclusion $(*)$ above, as illustrated by Fig [2], Corollary 3 in this paper combining with the work in 26 provides analogous results for linear temporal logical control of control systems with disturbances. In detail, Pola and Tabuada construct finite abstractions of control systems that are $A \varepsilon A$ bisimilar to the samples of control systems [26], while we demonstrate that if finite abstraction satisfies a specification $\phi$ under control then so does the samples for a looser specification $\operatorname{Tr}_{\varepsilon}(\phi)$ (see Corollary 3). These results inspire us to provide an approach for the design of control system as shown in Fig 2 first, construct finite abstraction that is $A \varepsilon A$ bisimilar to the sample of control system; second, find a strategy of finite abstraction enforcing the given $\mathrm{LTL}_{+}$specification $\phi$; and finally, construct controller for control systems based on this strategy so that sampling system satisfies the transformed specification $\operatorname{Tr}_{\varepsilon}(\varphi) 6$. The first step has been completed by Pola and Tabuada [26]. For the second step, an algorithm has been offered to find strategies of alternating transition systems enforcing linear temporal logical specifications [20] and this algorithm can be adopted to obtain the desired strategies for finite abstractions. So there is only one question left to answer: how to construct the desired controller for control system based on the strategy of finite abstraction. Our future work will focus on this issue.

\footnotetext{
5 In this figure, ATS is the abbreviation of alternating transition system.

6 Since we often just can observe the sampling systems rather than control systems with disturbances, it may be reasonable to require that the samples satisfy specifications under such controllers.
} 


\section{Conclusion}

This paper provides a modal characterization of alternating approximate bisimilarity. Since alternating approximate bisimilarity is not always an equivalence relation, its modal characterization can not be provided in the usual style. This paper introduces two relations over temporal logic $\mathrm{ATL}_{\varepsilon}$ and adopt these relations to establish the desired modal characterization of alternating approximate bisimilarity in a new style (see Theorem 22). This result reveals a relationship between the approximate equivalence among alternating transition systems and the temporal logical properties satisfied by these systems.

Pola and Tabuada adopt alternating transition systems to model the samples of control systems with disturbances and their finite abstractions, and introduce the notion of alternating approximate bisimilarity to capture the equivalence between these systems 25]26. Based on the modal characterization of alternating approximate bisimilarity obtained in this paper, we provide the transformation function $\operatorname{Tr}_{\varepsilon}$ from $\mathrm{LTL}_{+}$-specifications to $\mathrm{LTL}_{+}^{\varepsilon}$ specifications. Moreover, we show that, given a control system with disturbances, whose sampling system and finite abstraction are alternating approximate bisimilar, if the later realizes $\mathrm{LTL}_{+}$-specification $\phi$ under control, then the former satisfies the corresponding $\operatorname{LTL}_{+}^{\varepsilon}$-specification $\operatorname{Tr}_{\varepsilon}(\phi)$ under control. As illustrated in Fig 2, this result may be useful in designing the controller for control systems with disturbances. Future work will be devoted to perfecting the approach shown in Fig 2 .

\section{References}

1. Aczel, P., Mendler, N., (1989). A final coalgebra theorem. In: Proceedings. Category Theory and Computer Science, pp. 357-365. Springer

2. Alur, R., Henzinger, T., Kupferman, O. (2002). Alternating-time temporal logic. Journal of the ACM (JACM) 49(5): 672-713

3. Alur, R., Henzinger, T., Kupferman, O., Vardi, M., (1998). Alternating refinement relations. In: Proceedings. International Conference on Concurrency Theory (CONCUR), pp. 165-178. Springer

4. Alur, R., Henzinger, T., Lafferriere, G., Pappas, G. (2000). Discrete abstractions of hybrid systems. Proceedings of the IEEE 88(7): 971-984

5. van Breugel, F., (2005). A behavioural pseudometric for metric labelled transition systems. In: Proceedings. International Conference on Concurrency Theory (CONCUR), pp. 141-155. Springer

6. van Breugel, F., Sharma, B., Worrell, J. (2008). Approximating a behavioural pseudometric without discount for probabilistic systems. Logical Methods in Computer Science 4(2:2): $1-23$

7. van Breugel, F., Worrell, J. (2005). A behavioural pseudometric for probabilistic transition systems. Theoretical Computer Science 331(1): 115-142

8. Cámara, J., Girard, A., Gössler, G., (2011). Synthesis of switching controllers using approximately bisimilar multiscale abstractions. In: Proceedings. the 14th International Conference on Hybrid systems: Computation and Control, pp. 191-200. ACM

9. De Alfaro, L., Faella, M., Stoelinga, M. (2009). Linear and branching system metrics. IEEE Transactions on Software Engineering 35(2): 258-273

10. Desharnais, J.: Labelled markov processes. Ph.D. thesis, McGill University, (1999)

11. Desharnais, J., Gupta, V., Jagadeesan, R., Panangaden, P. (2004). Metrics for labelled markov processes. Theoretical Computer Science 318(3): 323-354 
12. Fainekos, G., Kress-Gazit, H., Pappas, G., (2005). Hybrid controllers for path planning: A temporal logic approach. In: Proceedings. the 44th IEEE Conference on Decision and Control, 2005 and 2005 European Control Conference. CDC-ECC'05, pp. 4885-4890. IEEE

13. Giacalone A. Jou C., S.S., (1990). Algebraic reasoning for probabilistic concurrent systems. In: Proceedings. IFIPWG 2.2/2.3Working Conf. on Programming Concepts and Methods, Sea of Gallilee, pp. 443-458. North-Holland

14. Girard, A., (2007). Approximately bisimilar finite abstractions of stable linear systems. In: Proceedings. Hybrid Systems: Computation and Control, pp. 231-244. IEEE

15. Girard, A., Pappas, G. (2007). Approximation metrics for discrete and continuous systems. IEEE Transactions on Automatic Control 52(5): 782-798

16. Girard, A., Pappas, G. (2009). Hierarchical control system design using approximate simulation. Automatica 45(2): 566-571

17. Girard, A., Pappas, G. (2011). Approximate bisimulation: A bridge between computer science and control theory. European Journal of Control 17(5): 568-578

18. Hennessy, M., Milner, R. (1985). Algebraic laws for nondeterminism and concurrency. Journal of the ACM (JACM) 32(1): 137-161

19. König, D.: Theorie der endlichen und unendlichen Graphen: Kombinatorische Topologie der Strekenkomplexe. (1936)

20. Kloetzer, M., Belta, C., (2008). Dealing with nondeterminism in symbolic control. In: Proceedings. Hybrid Systems: Computation and Control, pp. 287-300. Springer

21. Kloetzer, M., Belta, C. (2008). A fully automated framework for control of linear systems from temporal logic specifications. IEEE Transactions on Automatic Control 53(1): 287-297

22. Larsen, K., Yi, W., (1994). Time abstracted bisimulation: Implicit specifications and decidability. In: Proceeding. Mathematical Foundations of Programming Semantics, pp. 160-176. Springer

23. Park, D., (1981). Concurrency and automata on infinite sequences. In: Proceedings. the 5th GI-Conference on Theoretical Computer Science, pp. 167-183. Springer

24. Pola, G., Girard, A., Tabuada, P. (2008). Approximately bisimilar symbolic models for nonlinear control systems. Automatica 44(10): 2508-2516

25. Pola, G., Tabuada, P., (2008). Symbolic models for nonlinear control systems affected by disturbances. In: Proceedings. 47th IEEE Conference on Decision and Control, pp. 251-256. IEEE

26. Pola, G., Tabuada, P. (2009). Symbolic models for nonlinear control systems: Alternating approximate bisimulations. SIAM Journal on Control and Optimization 48 719-733

27. Tabuada, P. (2008). An approximate simulation approach to symbolic control. IEEE Transactions on Automatic Control 53(6): 1406-1418

28. Tabuada, P., Pappas, G., (2003). Finite bisimulations of controllable linear systems. In: Proceedings. 42nd IEEE Conference on Decision and Control, 2003., vol. 1, pp. 634-639. IEEE

29. Tabuada, P., Pappas, G., (2003). From discrete specifications to hybrid control. In: Proceedings. 42nd IEEE Conference on Decision and Control, 2003., vol. 4, pp. 33663371. IEEE

30. Tabuada, P., Pappas, G. (2006). Linear time logic control of discrete-time linear systems. IEEE Transactions on Automatic Control 51(12): 1862-1877

31. Wang, Y., (1990). Real-time behaviour of asynchronous agents. In: Proceedings. International Conference on Concurrency Theory (CONCUR), pp. 502-520. Springer

32. Ying, M. (2002). Bisimulation indexes and their applications. Theoretical Computer Science 275(1-2): 1-68

33. Ying, M. (2005). $\pi$-calculus with noisy channels. Acta Informatica 41(9): 525-593

34. Ying, M., Feng, Y., Duan, R., Ji, Z. (2009). An algebra of quantum processes. ACM Transactions on Computational Logic (TOCL) 10(3): 1-36

35. Zhang, J., Zhu, Z. (2007). A modal characterization of $\lambda$-bisimilarity. Int J Software Informatics 1(1): 85-99

36. Zhang, J., Zhu, Z. (2008). A behavioural pseudometric based on $\lambda$-bisimilarity. Electronic Notes in Theoretical Computer Science 220(3): 115-127 
37. Zhang, J., Zhu, Z. (2008). Characterize branching distance in terms of $(\eta, \alpha)$-bisimilarity Information and Computation 206(8): 953-965

38. Zhou, C., Ying, M. (2012). Approximating markov processes through filtration. Theoretical Computer Science 446: 75-97 